JOURNAL OF THE

AMERICAN MATHEMATICAL SOCIETY

Volume 16, Number 3, Pages 537-579

S 0894-0347(03)00423-5

Article electronically published on February 27, 2003

\title{
RESULTANTS AND CHOW FORMS VIA EXTERIOR SYZYGIES
}

\author{
DAVID EISENBUD, FRANK-OLAF SCHREYER, AND APPENDIX BY JERZY WEYMAN
}

Let $W$ be a vector space of dimension $n+1$ over a field $K$. The Chow divisor of a $k$-dimensional variety $X$ in $\mathbf{P}^{n}=\mathbf{P}(W)$ is the hypersurface, in the Grassmannian $\mathbf{G}_{k+1}$ of planes of codimension $k+1$ in $\mathbf{P}^{n}$, whose points (over the algebraic closure of $K$ ) are the planes that meet $X$. The Chow form of $X$ is the defining equation of the Chow divisor. For example, the resultant of $k+1$ forms of degree $e$ in $k+1$ variables is the Chow form of $\mathbf{P}^{k}$ embedded by the $e$-th Veronese mapping in $\mathbf{P}^{n}$ with $n=\left(\begin{array}{c}k+e \\ k\end{array}\right)-1$. More generally, the Chow divisor of a $k$-cycle $\sum_{i} n_{i}\left[V_{i}\right]$ on projective space is defined to be $\sum_{i} n_{i} D_{i}$, where $D_{i}$ is the Chow divisor of $V_{i}$. The Chow divisor of a sheaf $\mathcal{F}$ with $k$-dimensional support is the Chow divisor of the associated $k$-cycle of $\mathcal{F}$.

In this paper we will give a new expression for the Chow divisor and apply it to give explicit formulas in many new cases. Starting with a sheaf $\mathcal{F}$ on $\mathbf{P}^{n}$, we use exterior algebra methods to define a canonical and effectively computable Chow complex of $\mathcal{F}$ on each Grassmannian of planes in $\mathbf{P}^{n}$. If $\mathcal{F}$ has $k$-dimensional support, we show that the Chow form of $\mathcal{F}$ is the determinant of the Chow complex of $\mathcal{F}$ on the Grassmannian of planes of codimension $k+1$. The Beilinson monad of $\mathcal{F}$ Beilinson 1978 is the Chow complex of $\mathcal{F}$ on the Grassmannian of 0-planes (that is, on $\mathbf{P}^{n}$ itself.)

In particular, we are able to give explicit determinantal and Pfaffian formulas for resultants in some cases where no polynomial formulas were known. For example, the Horrocks-Mumford bundle gives rise to polynomial formulas for the resultant of five homogeneous forms of degrees 4,6 or 8 in five variables. The easiest of our new formulas to write down is for the resultant of 3 quadratic forms in three variables, the Chow form of the Veronese surface in $\mathbf{P}^{5}$. Using the tangent bundle of $\mathbf{P}^{2}$, conclude that it can be written in "Bézout form" (described below) as the Pfaffian of the matrix

$$
\left(\begin{array}{cccccccc}
0 & {[245]} & {[345]} & {[135]} & {[045]} & {[035]} & {[145]} & {[235]} \\
-[245] & 0 & -[235] & {[035]} & {[025]} & {[015]} & {[125]} & -[125]+[045] \\
-[345] & {[235]} & 0 & {[134]} & {[035]} & {[034]} & {[135]} & {[234]} \\
-[135] & -[035] & -[134] & 0 & {[023]} & {[013]} & {[123]-[034]} & -[123] \\
-[045] & -[025] & -[035] & -[023] & 0 & {[012]} & -[015] & -[024]+[015] \\
-[035] & -[015] & -[034] & -[013] & -[012] & 0 & {[023]-[014]} & -[023] \\
-[145] & -[125] & -[135] & -[123]+[034] & {[015]} & -[023]+[014] & 0 & -[124]+[035] \\
-[235] & {[125]-[045]} & -[234] & {[123]} & {[024]-[015]} & {[023]} & {[124]-[035]} & 0
\end{array}\right)
$$

Received by the editors November 16, 2001.

2000 Mathematics Subject Classification. Primary 13P05, 14Q99; Secondary 13D25, 14 F05.

Key words and phrases. Chow form, resultants, Beilinson monad, Ulrich modules. 
where the brackets $[i j k]$ denote the corresponding Plücker coordinates of the space spanned by the three given quadratic forms with respect to the ordered basis $x^{2}, x y, x z, y^{2}, y z, z^{2}$ for the space of all quadratic forms.

There are (at least) two types of formulas for resultants or Chow forms:

Bézout formulas for resultants. The classic formula of Bézout (see, for example, Gel'fand et al. 1994, Chapter 12, (1.17) and (1.18)]) gives the resultant of two homogeneous forms in two variables as a determinant of linear forms in the Plücker coordinates of the space generated by the two forms. We will call any formula for the Chow form in Plücker coordinates a Bézout expression. Our simplest new Bézout expression is for the resultant of three quadratic forms in three variables: it is the Pfaffian (三 square root of the determinant) of the alternating matrix of linear forms in the Plücker coordinates. Using the theory of rank two vector bundles on $\mathbf{P}^{2}$, we can construct Bézout formulas for forms in three variables of any degree. In fact, we construct continuous families of such formulas.

Stiefel formulas for resultants. The Grassmannian is a quotient of an open set in the variety of $(k+1) \times(n+1)$ matrices over $K$; the entries of these matrices are called Stiefel coordinates on the Grassmannian (or on the Stiefel manifold). Pulling back the Chow divisor, we get a divisor whose ideal is generated by a polynomial in the Stiefel coordinates. For example, if $X$ is the rational normal curve, this polynomial is the Sylvester determinant. Even when we cannot express the Chow form of a variety as the determinant or Pfaffian of a matrix in the Plücker coordinates, we can sometimes express it as the determinant or Pfaffian of a map of equivariant vector bundles on the Grassmannian. Such maps pull back to matrices in the Stiefel coordinates whose determinant or Pfaffian defines the (closure of the) preimage of the Chow divisor. We say that such a matrix gives a Stiefel expression for the Chow form. The classical Sylvester determinant is such a Stiefel expression.

Explicit Stiefel expressions for the resultant of $k+1$ forms of degree $d \geq 2$ in $k+1$ variables (Chow form of the $d$-uple embedding of $\mathbf{P}^{k}$ ) have been known for $k \leq 3$ (all $d$ ) and $k=4, d=2,3$ and $k=5, d=2$ (see, for example, Gel'fand et al. 1994, Chapter 13, Prop. 1.6], [D'Andrea and Dickenstein 2001. Using our method and constructions of vector bundles on $\mathbf{P}^{k}$, we give new Stiefel expressions. In particular, the Horrocks-Mumford bundle gives rise to Pfaffian Stiefel expressions for the resultants of 5 forms of degrees 4,6 , or 8 in 5 variables. The matrices involved are too large to exhibit here; but Macaulay2 programs for producing them and other new examples can be found at http://msri.org/people/staff/de/ ChowM2scripts.

WhAT IS THE CHOW DIVISOR OF A SHEAF?

Let

$$
\mathbf{P}^{n} \stackrel{\pi_{1}}{\longleftarrow} \mathbf{F}_{l} \stackrel{\pi_{2}}{\longrightarrow} \mathbf{G}_{l}
$$

be the incidence correspondence; that is, let

$$
\mathbf{F}_{l}=\left\{(p, L) \in \mathbf{P}^{n} \times \mathbf{G}_{l} \mid p \in L\right\} .
$$

In the case $l=k+1$, the Chow divisor of a $k$-dimensional subvariety $X \subset \mathbf{P}^{n}$ is by definition $D_{X}=\pi_{2}\left(\pi_{1}^{-1} X\right)$; one can check immediately that this is a divisor in $\mathbf{G}_{l}$. 
More generally if $\mathcal{F}$ is any sheaf on $\mathbf{P}^{n}$ whose support $Y$ has dimension $k$, then the $k$-cycle associated to $\mathcal{F}$ is

$$
\sum_{\operatorname{dim} X=k} \underset{\mathcal{O}_{\mathbf{P}^{n}, X}}{\operatorname{length}}\left(\mathcal{O}_{\mathbf{P}^{n}, X} \otimes \mathcal{F}\right) \cdot[X]
$$

where the sum is taken over all $k$-dimensional subvarieties of $\mathbf{P}^{n}$ (or equivalently over the $k$-dimensional components of $Y$ ). The Chow divisor of $\mathcal{F}$ is defined to be the corresponding sum of Chow divisors

$$
\sum_{\operatorname{dim} X=k} \underset{\mathcal{O}_{\mathbf{P}^{n}, X}}{\operatorname{length}}\left(\mathcal{O}_{\mathbf{P}^{n}, X} \otimes \mathcal{F}\right) \cdot D_{X}
$$

For example, if $X \subset \mathbf{P}^{n}$ is any subvariety of dimension $k$ and if $\mathcal{F}=\mathcal{O}_{X}$ (or any line bundle on $X$ ), then the Chow divisor of $\mathcal{F}$ is the Chow divisor of $X$; more generally if $\mathcal{F}$ is a vector bundle of rank $r$ on the $X$, then the Chow divisor of $\mathcal{F}$ is $r$ times that of $X$.

Since the generic plane of codimension $k+1$ meeting a component $X$ of $Y=$ $\operatorname{Supp} \mathcal{F}$ meets $X$ in just one general point of $X$, we see (in the case $l=k+1$ ) that $\mathcal{G}=\left(\pi_{2}\right)_{*} \pi_{1}^{*} \mathcal{F}$ is supported precisely on the set $\pi_{2} \pi_{1}^{-1} Y$. The same argument shows that the generic rank of $\mathcal{G}$ on $D_{X}$ is length $\operatorname{O}_{\mathbf{P}^{n}, X} \mathcal{O}_{\mathbf{P}^{n}, X} \otimes \mathcal{F}$. Thus the Chow divisor of $\mathcal{F}$ is actually the divisor associated to the sheaf $\mathcal{G}$ on $\mathbf{G}_{l}$.

Finding the divisor associated to a sheaf. To make use of this idea, we need to be able to go from the sheaf $\mathcal{G}$, supported in codimension 1, to a description of the divisor that is its support. A divisor is best described as a line bundle and a global section of that line bundle. Any line bundle on the Grassmannian is a power of the hyperplane bundle, so the divisor can be represented simply as a polynomial in the Plücker coordinates, the Chow form. However, the sheaf $\mathcal{G}$ itself, from which we must start, may be very complicated. For example, it may have high projective dimension and embedded components.

Consider for a moment the general problem of computing the divisor associated to a sheaf $\mathcal{G}$ with codimension 1 support on a smooth variety $Z$. We suppose that $\mathcal{G}$ is presented as the cokernel of a map $\phi: A \rightarrow B$ of vector bundles, and we wish to find - as explicitly as possible - a line bundle on $Z$ and a global section of it whose divisor is the divisor of $\mathcal{F}$.

Let $b$ be the rank of $B$. The $b$-th exterior power of $\phi$ is a map $\bigwedge^{b} \phi: \bigwedge^{t} A \rightarrow \bigwedge^{b} B$, which gives rise to a map $\bigwedge^{b} A \otimes \bigwedge^{b} B^{*} \rightarrow \mathcal{O}_{Z}$. The zero-th Fitting ideal $\operatorname{Fitt}_{0}(\mathcal{G})$ of $\mathcal{G}$ is by definition the image of this map. The divisor associated to $\mathcal{G}$ is the same as the divisor associated to $\mathcal{O}_{Z} / \operatorname{Fitt}_{0}(\mathcal{G})$. (This may be proved by localizing at a prime of codimension 1 and then using [Fulton 1984, Example A.2.3].) Since $\mathcal{G}$ has codimension 1 support we must have $\operatorname{rank} A \geq \operatorname{rank} B$. In case $\operatorname{rank} A=\operatorname{rank} B$, the desired line bundle is $\bigwedge^{b} A^{*} \otimes \bigwedge^{b} B$ and the dual of the map $\bigwedge^{b} A \otimes \bigwedge^{b} B^{*} \rightarrow \mathcal{O}_{Z}$ sends $1 \in \mathcal{O}_{Z}$ to the desired global section. This means that the divisor of $\mathcal{G}$ is defined by the determinant of $\phi$. One of the central goals of this paper, in the setting of Chow forms, is to give a simple characterization of some sheaves $\mathcal{F}$ for which the corresponding sheaf $\mathcal{G}$ on the Grassmannian is naturally presented by a map between vector bundles of the same rank or has a presentation by a map represented by a square matrix of linear forms. These are the "weakly Ulrich sheaves" and "Ulrich sheaves" described below. 
When $\operatorname{rank} A>\operatorname{rank} B$, the situation is much more complicated. The desired divisor is defined locally by the greatest common divisor of the $b \times b$ minors of a matrix representing $\phi$, but this is much less explicit than the description above.

A better generalization of the case $\operatorname{rank} A=\operatorname{rank} B$ was discovered (in a special case) by Arthur Cayley Cayley 1848 and greatly generalized by Grothendieck in an unpublished letter to David Mumford in 1962; the details are worked out in Knudsen and Mumford 1976, where the letter is described. In brief, the divisor of $\mathcal{F}$ is the determinant divisor of any finite complex $\mathcal{C}$ of vector bundles whose homology differs from $\mathcal{G}$ only in codimension $\geq 2$. In the local case the determinant of $\mathcal{C}$ may be represented as a rational function, the alternating product of certain minors in matrices representing the differentials of $\mathcal{C}$. A good introduction to part of the Cayley-Grothendieck theory can be found in the Appendix A of Gel'fand et al. 1994.

Chow complexes. Let us now return to the setting of the Chow form, and take $Z=\mathbf{G}_{l}$, with $l=k+1$ and $\mathcal{G}=\left(\pi_{2}\right)_{*} \pi_{1}^{*} \mathcal{F}$. Cayley studied the case where $X$ is the $d$-th Veronese embedding of $\mathbf{P}^{k}$ and $\mathcal{F}$ is a sufficiently positive line bundle on $\mathbf{P}^{k}$. He produced an explicit free resolution of $\mathcal{G}$ to play the role of the complex $\mathcal{C}$ above. His constructions were studied and generalized by Macaulay, Jouanolou and other authors who derived in this way expressions for resultants as rational functions in the Plücker or Stiefel coordinates. For modern results, see Weyman and Zelevinsky 1994], Jouanolou 1995]. An exposition may be found in Gel'fand et al. 1994. Of course the Chow form is a polynomial: in these rational function expressions the denominator divides the numerator. However, it is not known how to make the quotient explicit. Refinements aimed at reducing the degree of the denominator are an active subject of research; see, for example, D'Andrea and Dickenstein 2001] and [D'Andrea 2002].

Grothendieck extended Cayley's theory to apply to any sheaf $\mathcal{F}$. He observed that there exists a locally free complex $\mathcal{C}$, well-defined up to quasi-isomorphism, with

$$
\mathcal{C} \simeq \mathbf{R} \pi_{2 *}\left(\pi_{1}^{*} \mathcal{F}\right)
$$

and $\mathrm{H}^{0} \mathcal{C} \cong \mathcal{G}$ while $\mathrm{H}^{i} \mathcal{C}$ is isomorphic to the higher direct image $\mathbf{R} \pi_{2 *}\left(\pi_{1}^{*} \mathcal{F}\right)$, which is supported in codimension $\geq 2$ in $\mathbf{G}$. Thus the Chow divisor of $\mathcal{F}$ is the divisor of the determinant of $\mathcal{C}$ (in Cayley's case all the higher direct images are 0 ). The problem with Grothendieck's idea is that for general $\mathcal{F}$ it has not been possible until now to give an effectively computable complex $\mathcal{C} \simeq \mathbf{R} \pi_{2 *}\left(\pi_{1}^{*} \mathcal{F}\right)$. However, the determinant is so robust that it can be computed from the associated graded complex of a filtered complex representing $\mathbf{R} \pi_{2 *}\left(\pi_{1}^{*} \mathcal{F}\right)$; such complexes can sometimes be computed from spectral sequences (the construction of the Chow form as the "determinant of a spectral sequence" by Weyman and Zelevinsky, described in Gel'fand et al. 1994, Section 3.4.C], is such a computation).

\section{Main Results}

This paper divides naturally into two parts. In Sections 1-3 we treat the general theory of Chow complexes and (weakly) Ulrich sheaves. In Sections 4-6 we deal with various families of examples and with the question of the existence of (weakly) Ulrich sheaves in these examples. 
Describing a Chow complex. Our first main result gives a canonical Chow complex

$$
\mathbf{U}_{k+1}(\mathcal{F}) \simeq \mathbf{R} \pi_{2 *}\left(\pi_{1}^{*} \mathcal{F}\right)
$$

for each coherent sheaf $\mathcal{F}$, part of a sequence of complexes generalizing the Beilinson monad for $\mathcal{F}$. The construction is so explicit that it can be made on a computer. Recall that a plane of any codimension $l$ in $\mathbf{P}^{n}$ corresponds to an $(n+1-l)$-quotient of $W$, and thus to an $l$-dimensional subspace of $W$. We write $U_{l}$ for the tautological $l$-subbundle on $\mathbf{G}_{l}$.

Theorem 0.1. For any coherent sheaf $\mathcal{F}$ on $\mathbf{P}^{n}$ and any $0 \leq l \leq n$ there is a canonical complex $\mathbf{U}_{l}(\mathcal{F})$ of vector bundles on $\mathbf{G}_{l}$ with

$$
\mathbf{U}_{l}(\mathcal{F}) \simeq \mathbf{R} \pi_{2 *}\left(\pi_{1}^{*} \mathcal{F}\right) .
$$

The e-th term of $\mathbf{U}_{l}(\mathcal{F})$ is $\sum_{j} \mathrm{H}^{j}(\mathcal{F}(e-j)) \otimes \bigwedge^{j-e} U_{l}$.

The complex $\mathbf{U}_{n}(\mathcal{F})$ is the Beilinson monad on $\mathbf{P}^{n}$ that is defined in Eisenbud et al. 2001. The sheaf $\mathcal{F}$ can be recovered from $\mathbf{U}_{n}(\mathcal{F})$ simply by taking homology. The sheaf $\mathcal{F}$ can be recovered from some of the other $\mathbf{U}_{l}(\mathcal{F})$ as well: Just as one can recover a variety of dimension $k$ from its Chow divisor in $\mathbf{G}_{k+1}$, so one can recover any sheaf $\mathcal{F}$ whose support has dimension at most $k$ from the Chow complex $\mathbf{U}_{l}(\mathcal{F})$ as long as $l>k$. All these matters are explained in Section 1 .

Most significant in our treatment is that we can give an explicit and canonical description of the maps in the complex $\mathbf{U}_{l}$. Until now, in general, it has only been possible to write down the sheaves in such a complex (see, for example, Gel'fand et al. 1994, Section 3.4E], "Weyman's complexes") or to approximate the maps via a spectral sequence. With enough vanishing of cohomology, it was possible to write down the maps; but these cases were often not the ones of primary interest. Also, previous authors seem only to have considered formulas coming from the case where $\mathcal{F}$ is a line bundle on its support. Our technique allows us to recover explicit expressions of the Chow form in all the previously known cases, and, using vector bundles as in the examples mentioned above, some new ones.

Finding simple Chow complexes. The most useful formulas for the Chow form occur when the complex $\mathbf{U}=\mathbf{U}_{k+1}(\mathcal{F})$ has just one nontrivial map $\Psi$ :

$$
\mathbf{U}: \cdots \rightarrow 0 \rightarrow 0 \rightarrow C^{-1} \stackrel{\Psi}{\longrightarrow} C^{0} \rightarrow 0 \rightarrow 0 \rightarrow \cdots .
$$

In this case the Chow form of $\mathcal{F}$ is given by the determinant of $\Psi$, and if the bundles $C^{i}$ are direct sums of exterior powers of the tautological bundles, then one gets a determinantal expression for the Chow form in Stiefel coordinates.

An even better case occurs when $C^{-1}$ and $C^{0}$ are direct sums of line bundles. Then the Chow form of $\mathcal{F}$ is given directly as a determinant in the Plücker coordinates - that is, we get a Bézout expression for a power of the Chow form of the support of $\mathcal{F}$. If $\mathcal{F}$ has rank 1 , or if $\mathcal{F}$ has rank 2 and the map $\Psi$ is skew-symmetric, so that we can extract the square root of the determinant as the Pfaffian, then we get the Chow form of the support of $\mathcal{F}$ itself.

Such cases are considered in Section 2. Our second main result describes precisely the conditions on the sheaf $\mathcal{F}$ that are necessary for the Chow complex $\mathbf{U}_{k+1}(\mathcal{F})$ to degenerate to one of these special forms. For example: 
Theorem 0.2. The Chow complex $\mathbf{U}_{k+1}(\mathcal{F})$ above degenerates to a single map $\mathcal{O}_{\mathbf{G}}^{d}(-1) \rightarrow \mathcal{O}_{\mathbf{G}}^{d}$ if and only if the module of twisted global sections $\bigoplus_{m} \mathrm{H}^{0}(\mathcal{F}(m))$ is a Cohen-Macaulay module with a linear free resolution.

Here by a linear free resolution we mean a free resolution over the polynomial ring $S=k\left[x_{0}, \ldots, x_{r}\right]$ of the form

$$
\cdots \rightarrow S^{r_{2}}(-2) \rightarrow S^{r_{1}}(-1) \rightarrow S^{r_{0}} .
$$

Cohen-Macaulay graded modules with linear free resolutions $M$ were studied by Bernd Ulrich under the name "maximally generated maximal Cohen-Macaulay modules" Ulrich 1984 and have been studied by others under the names "linear maximal Cohen-Macaulay modules" or "Ulrich modules"; see [Brennan et al. 1987], Backelin and Herzog, 1989] and the references given there. We shall call the corresponding sheaves Ulrich sheaves. For example, a line bundle $\mathcal{F}$ on a curve $X$ of genus $g$ embedded in $\mathbf{P}^{n}$ is an Ulrich sheaf if and only if $\mathcal{F}(-1)$ has degree $g-1$ and no global sections; that is, $\mathcal{F}(-1)$ corresponds to a point in $\operatorname{Pic}^{g-1}(X)$ which lies outside the theta divisor $\Theta \subset \operatorname{Pic}^{g-1}(X)$.

More generally we say that a sheaf $\mathcal{F}$ whose Chow complex $\mathbf{U}_{k+1}(\mathcal{F})$ has only two terms is weakly Ulrich, because the necessary cohomological vanishing is nearly the same as for Ulrich sheaves. For example, a Cohen-Macaulay module $M=$ $\bigoplus_{m} \mathrm{H}^{0}(\mathcal{F}(m))$ with $\mathrm{H}^{0}(\mathcal{F}(-1))=0$ and $\mathrm{H}^{0}(\mathcal{F}) \neq 0$ corresponds to a weakly Ulrich sheaf if and only if $M$ is 1-regular.

Duality and Pfaffian expressions. In Section 3 we turn to the problem of giving determinantal and Pfaffian expression for the Chow form of an Ulrich sheaf $\mathcal{F}$. We can express them directly in terms of the free resolution of the corresponding module $M$ by using a construction developed in Angéniol and Lejeune-Jalabert 1989 to describe Atiyah classes. Suppose that

$$
0 \rightarrow F_{c} \stackrel{\phi_{c}}{\longrightarrow} \cdots \rightarrow F_{1} \stackrel{\phi_{1}}{\longrightarrow} F_{0}
$$

is a linear free resolution of $M$ as above. Regarding the $\phi_{i}$ as matrices of elements of $W$, we can compose them as if they were matrices of linear forms in the exterior algebra: we write $\Psi_{\mathcal{F}}:=(1 / c !) \phi_{1} \wedge \phi_{2} \wedge \cdots \wedge \phi_{c}$ for this product (defined in a slightly different way in positive characteristic), which is represented by a matrix of forms in $\bigwedge^{c} W$. We may identify $\bigwedge^{c} W$ with the the space of linear forms on $\mathbf{G}_{k+1}$ and we have:

Theorem 0.3. If $\mathcal{F}$ is an Ulrich sheaf, then $\Psi_{\mathcal{F}}$ is the (only) nonzero map in the Chow complex $\mathbf{U}_{k+1}(\mathcal{F})$. In particular the Chow form of $\mathcal{F}$ is $\operatorname{det} \Psi_{\mathcal{F}}$. If $\mathcal{F}$ is a vector bundle on a $k$-dimensional variety $X$, and $\mathcal{F}$ is skew-symmetric in an appropriate sense, then (in characteristic not 2) $\Psi_{\mathcal{F}}$ is skew-symmetric, and the square-root of the Chow form of $\mathcal{F}$ is the Pfaffian of $\Psi_{\mathcal{F}}$.

Theorem 0.3 gives a new method for constructing resultants and Chow forms: find Ulrich sheaves (or weakly Ulrich sheaves or Ulrich sheaves satisfying the skewsymmetry condition...) and then construct the map $\Psi_{\mathcal{F}}$. Given $\mathcal{F}$, we derive two ways to do this. Most practical for most explicit computations is the method illustrated in Section 4 in the classical case of binary forms as well as other situations, including doubly periodic functions (it is also the method used for toric surfaces in Khetan 2002]): one computes the multiplication map on global sections, $W \otimes H^{0} \mathcal{F}(n) \rightarrow \mathcal{F}(n+1)$, forms from it a map of free modules over the exterior 
algebra, and takes a certain syzygy matrix of the kernel of this map. On the other hand, we get somewhat more theoretical control from the product formula above; for example, we need this to prove Theorem 0.3 .

Explicit computations. Sections 4-6 of this paper treat a number of examples. We can be completely explicit in only a few cases, and these sections leave open a multitude of theoretical and practical problems. Of greatest importance is this:

Problem. Is every variety (or even scheme) $X \subset \mathbf{P}^{n}$ the support of an Ulrich sheaf? If so, what is the smallest possible rank for such a sheaf?

For example, Brennan, Herzog, and Ulrich showed that when $X$ is an arithmetically Cohen-Macaulay curve over an infinite field, or a a complete intersection, or a linear determinantal variety, then $X$ has an Ulrich sheaf Brennan et al. 1987. and Backelin and Herzog. In case $X$ is the hypersurface $F=0$, for example, they construct an Ulrich sheaf whose rank is exponential in the number of monomials required to express $F$. For a regular quadric hypersurface in $\mathbf{P}^{r}$ the situation is completely understood: There [Buchweitz Eisenbud and Herzog 1987] (see also Swan 1985) show that the minimal rank of an Ulrich module is precisely $2^{\lfloor(r) / 2\rfloor-1}$. Also, in the case of plane curves over an algebraically closed field, Ulrich modules of rank 1 always exist (see below.)

Turning to Veronese embeddings of projective spaces, Doug Hanes showed in his thesis under Hochster that the $d$-uple embeddings of $\mathbf{P}^{k}$ have Ulrich sheaves when $k \leq 2$ or $k=3$ and $d=2^{r}$ is a power of two Hanes 2000. We prove a number of new existence results, which we now summarize.

Curves. Section 4 is devoted to the case of curves. We complete (and reprove) the result of Brennan, Ulrich, and Herzog by showing that, if the ground field is infinite, every curve $X \subset \mathbf{P}^{n}$ has skew-symmetric rank 2 Ulrich sheaves. If the field is algebraically closed, there are rank 1 Ulrich sheaves; they are in oneto-one correspondence with the line bundles of degree $g-1$ on $X$ that have no sections. Thus there are Bézout expressions for the Chow forms of such curves. This generalizes the case of binary forms, in which $X=\mathbf{P}^{1}$ and the line bundle is $\mathcal{O}_{\mathbf{P}^{1}}(-1)$. It also generalizes the well-known result that the equation of any plane curve over an algebraically closed field can be written as the determinant of a matrix of linear forms; see for example [Vinnikov 1989, Beauville 2000].

Such Ulrich sheaves give rise, in principle, to continuous families of resultant formulas for the sections of any very ample line bundle on a curve of genus $\geq 1$, but it is not easy to make such formulas explicit. We illustrate with the case of hyperelliptic curves and provide a resultant formula for functions of the form $a+b \sqrt{f}, \quad c+d \sqrt{f}$ where $a, b, c, d$ and $f$ are polynomials in one variable. We carry out the proof completely only in case the degrees of the various polynomials are small. In the special case of elliptic curves, we get a resultant formula for doubly periodic functions written in terms of the Weierstrass $\wp$-function and its derivative.

Projective spaces: New resultant formulas. In Section 5 we turn to the case where $X \subset \mathbf{P}^{n}$ is the $d$-th Veronese ( $d$-uple) embedding of $X=\mathbf{P}^{k}$. This is the case that gives rise to resultant formulas for $k+1$ forms of degree $d$ in $k+1$ variables. We give cohomological criteria for a bundle on $\mathbf{P}^{k}$ to be Ulrich for the $d$-uple embedding. Following a suggestion of Jerzy Weyman, we use this to extend Hanes' results and show that every Veronese variety has an Ulrich sheaf, obtained 
by applying a certain (unique) Schur functor to the tautological quotient bundle. This gives a way of writing a power of the resultant as the determinant of a matrix of linear forms in the Plücker coordinates. This might be useful for computation: to determine whether or not a set of polynomials has a common zero, a power of the resultant is just as good as the resultant itself.

In Section 5 we also find (many) Ulrich modules of rank 2 for each Veronese embedding of $\mathbf{P}^{2}$. We prove a lower bound on the ranks of possible Ulrich modules and using this and a result of Hartshorne-Hirschowitz on the existence of mathematical instanton bundles, we show that rank 2 Ulrich modules exist on the $d$-uple embedding of $\mathbf{P}^{3}$ if and only if $d$ is not divisible by 3 . On $\mathbf{P}^{4}$ we show that the Horrocks-Mumford bundle is weakly Ulrich for the 4, 6, and 8-uple embeddings and satisfies the skew-symmetry condition necessary for us to get a Pfaffian Stiefel formula for the corresponding resultants.

Surfaces. Section 6 is concerned with the existence of skew-symmetric rank 2 Ulrich sheaves on various surfaces and thus with Pfaffian resultant formulas generalizing the Bézout formula for $\mathbf{P}^{2}$ given at the beginning of this introduction. We use Mukai's construction of vector bundles on surfaces and describe the necessary data. Our main result is the existence of skew-symmetric rank 2 Ulrich modules for certain embeddings of the plane blown up at a set of points. These modules lead to Pfaffian Bézout expressions for the resultant of 3 ternary forms of degree $d$ with assigned simple base points, valid when the ideal defining the set of base points is generated in degree $<d$.

Maps of tautological sheaves. Throughout this paper we rely on a certain construction of homomorphisms between exterior powers of the tautological bundle on a Grassmannian, explained in Section 1 In the Appendix Jerzy Weyman proves - in all characteristics - that in fact every homomorphism arises from this construction.

\section{Chow Complexes obtained from the Beilinson monad}

As above we write $\mathbf{G}_{l}$ for the Grassmannian of planes of codimension $l$ in $\mathbf{P}:=$ $\mathbf{P}^{n}=\mathbf{P}(W)$ and $\mathbf{F}_{l}$ for the flag variety of flags consisting of a point $p \in \mathbf{P}$ and a plane $L \in \mathbf{G}_{l}$ of codimension $l$ in $\mathbf{P}$ containing $p$. Throughout this section we will consider the incidence correspondence

$$
\mathbf{P} \stackrel{\pi_{1}}{\longleftarrow} \mathbf{F}_{l} \stackrel{\pi_{2}}{\longrightarrow} \mathbf{G}_{l}
$$

Let $0 \rightarrow U \rightarrow W \otimes \mathcal{O}_{\mathbf{G}_{l}} \rightarrow Q \rightarrow 0$ be the tautological sequence on the Grassmanian $\mathbf{G}_{l}$, so that $U=U_{l}$ is a bundle of rank $l$. We write $E$ for the exterior algebra $\bigwedge V$, where $V=W^{*}$. Any element $a \in \bigwedge^{p} V$ gives rise to a "contraction" mapping $a: \bigwedge^{q} U \rightarrow \bigwedge^{q-p} U$ as follows: First, $a$ induces a homomorphism $\bigwedge^{p} W \rightarrow K$ by the usual contraction, and thus a homomorphism of sheaves

$$
\bigwedge^{p} U \hookrightarrow \bigwedge^{p} W \otimes \mathcal{O}_{\mathbf{G}_{l}} \rightarrow \mathcal{O}_{\mathbf{G}_{l}}
$$

Using the diagonal map [Eisenbud 1995, A2.4, p. 582] $\bigwedge^{q} U \stackrel{\Delta_{U}}{\longrightarrow} \bigwedge^{q-p} U \otimes \bigwedge^{p} U$, we get the desired map

$$
\bigwedge^{q} U \stackrel{(1 \otimes a) \Delta_{U}}{\longrightarrow} \bigwedge^{q-p} U
$$


We use the well-known part (a) of the following lemma heavily. Part (b) is needed for Proposition 1.3.

Proposition 1.1. Let $U=U_{l}$ be the tautological subbundle on $\mathbf{G}_{l}$.

(a) The maps above make $\bigwedge U$ into a module over $\wedge V$.

(b) (J. Weyman) The maps

$$
\bigwedge^{p} V \rightarrow \operatorname{Hom}\left(\bigwedge^{q} U, \bigwedge^{q-p} U\right)
$$

are isomorphisms for all integers $p, q$ such that $0 \leq q-p, q \leq l$.

Proof. (a) With notation as above, the naturality of the diagonal maps shows that the diagrams

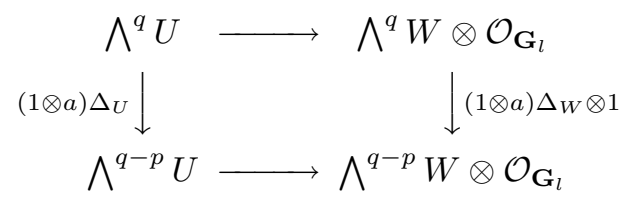

commute. Since $\bigwedge W$ is naturally a module over $E=\bigwedge V$ by this action (see for example [Eisenbud 1995, Appendix A2.4.1]), so is $\wedge U$.

(b) This is proved in an appendix to this paper by J. Weyman. In characteristic 0 the result follows from Bott's vanishing theorem [Jantzen 1987. In arbitrary characteristic it is more delicate.

We will grade $E$ by the convention that the elements of $V$ have degree -1 . As usual we write $E(q)$ for the free graded $E$-module of rank 1, with generator in degree $-q$. Thus, for example, if $q>p$, then $\operatorname{Hom}(E(q), E(q-p))=E_{-p}=\bigwedge^{p} V$. Recall from Eisenbud et al. 2001 that a Tate resolution is a doubly infinite exact complex of finitely generated free graded $E$-modules that is minimal, in the sense that each free module maps into $V$ times the next one. If $\mathcal{F}$ is any coherent sheaf on $\mathbf{P}$, then there is a Tate resolution $\mathbf{T}(\mathcal{F})$ naturally associated to $\mathcal{F}$, which can be computed, using free resolutions over an exterior algebra, from the module of twisted global sections $\bigoplus_{e} \mathrm{H}^{0} \mathcal{F}(e)$. Its $e$-th term is isomorphic to

$$
T^{e}(\mathcal{F})=\bigoplus_{j} \mathrm{H}^{j}(\mathcal{F}(e-j)) \otimes E(j-e) .
$$

For all this see [Eisenbud et al. 2001].

We can define the additive functor $\mathbf{U}_{l}$ from graded free modules over $E$ to locally free sheaves on $\mathbf{G}_{l}$ by sending $E(p)$ to $\mathbf{U}_{l}(E(p))=\bigwedge^{p} U$, where $U=U_{l}$ is the tautological subbundle, and sending a map $\eta: E(q) \rightarrow E(q-p)$ to the map $\mathbf{U}_{l}(\eta): \bigwedge^{q} U \rightarrow \bigwedge^{q-p} U$ made from the element $\bigwedge^{p} V$ corresponding to $\eta$. If $\mathbf{T}$ is any Tate resolution, then for $e \gg 0$ or $e \ll 0$ we have $\mathbf{U}_{l}\left(T^{e}\right)=0$, so $\mathbf{U}_{l}(\mathcal{F}):=\mathbf{U}_{l}(\mathbf{T})$ is a bounded complex of locally free sheaves on $\mathbf{G}_{l}$.

For example, $\mathbf{U}_{n}(\mathcal{F})$ is shown in Eisenbud et al. 2001] to be a Beilinson monad for the sheaf $\mathcal{F}$ in the sense that it has the terms above, and its only homology is $\mathcal{F}$, in degree 0 (the functor $\mathbf{U}_{n}$ is called $\Omega$ in that paper). Here is a generalization for all $l$.

Theorem 1.2. If $\mathcal{F}$ is a sheaf on $\mathbf{P}^{n}$, the complex $\mathbf{U}_{l}(\mathcal{F})$ represents $\mathbf{R} \pi_{2 *}\left(\pi_{1}^{*} \mathcal{F}\right)$ in the derived category of sheaves on the Grassmannian $\mathbf{G}_{l}$. 
Proof. By Eisenbud et al. 2001, Theorem 6.1], $\mathbf{U}_{n}(\mathcal{F})$ is a representative of $\mathcal{F}$ in $D^{b}\left(\operatorname{Coh}\left(\mathbf{P}^{n}\right)\right)$. We will show first that $\mathbf{U}_{l}(\mathcal{F})=\pi_{2 *}\left(\pi_{1}^{*} \mathbf{U}_{n}(\mathcal{F})\right)$, and second that $\mathbf{R}^{i} \pi_{2 *}\left(\pi_{1}^{*}\left(\bigwedge^{p} U_{n}\right)\right)=0$ for $i>0$. It follows that $\mathbf{R} \pi_{2 *} \pi_{1}^{*} \mathbf{U}_{n}(\mathcal{F}) \cong \pi_{2 *}\left(\pi_{1}^{*} \mathbf{U}_{n}(\mathcal{F})\right)=$ $\mathbf{U}_{l}(\mathcal{F})$, as desired.

On $\mathbf{F}$ we have inclusions of the universal subbundles

$$
\pi_{2}^{*}\left(U_{l}\right) \subset \pi_{1}^{*}\left(U_{n}\right) \subset W \otimes \mathcal{O}_{\mathbf{F}} .
$$

Pushing the left hand inclusion forward we get a canonical map $U_{l}=\pi_{2 *} \pi_{2}^{*} U_{l} \rightarrow$ $\pi_{2 *} \pi_{1}^{*} U_{n}$, and we deduce similar maps on the exterior powers. To show that these are isomorphisms, we may compute fiber by fiber. If $u \in \mathbf{G}_{l}$, then we will also write $u \subset W$ for the corresponding $l$-dimensional linear subspace.

Setting $\mathbf{P}^{\prime}=\mathbf{P}(W / u) \subset \mathbf{P}(W)$, we have the decomposition

$$
\left.\bigwedge^{p} U_{n}\right|_{\mathbf{P}^{\prime}} \cong \bigoplus_{i=0}^{p} \bigwedge^{i} u \otimes \bigwedge^{p-i} U_{n-l}^{\prime},
$$

where $U_{n-l}^{\prime}$ denotes the tautological subbundle on $\mathbf{P}^{\prime}$. Thus the map $\wedge^{p} u \rightarrow$ $\mathrm{H}^{0}\left(\bigwedge^{p} U_{n} \mid \mathbf{P}^{\prime}\right)$ is an isomorphism, and all other cohomology of $\bigwedge^{p} U_{n} \mid \mathbf{P}^{\prime}$ vanishes.

By base change [Hartshorne 1977, III.12] we see that $\mathbf{R}^{i} \pi_{2 *}\left(\pi_{1}^{*} \bigwedge^{p} U_{n}\right)=0$ for $i>0$ while $\pi_{2 *}\left(\pi_{1}^{*} \bigwedge^{p} U_{n}\right) \cong \bigwedge^{p} U_{l}$.

The sheaf $\mathcal{F}$ is determined from $\mathbf{U}_{n}(\mathcal{F})$, the Beilinson monad, by the formula $\mathcal{F}=\mathrm{H}^{0}\left(\mathbf{U}_{n}(\mathcal{F})\right)$. More generally, when $l>k$, we can still recover $\mathcal{F}$ from $\mathbf{U}_{l}(\mathcal{F})$.

Proposition 1.3. If $\mathcal{F}$ is a coherent sheaf of dimension $k$ on $\mathbf{P}$ and $l>k$, then $\mathcal{F}$ is determined by the complex $\mathbf{U}_{l}(\mathcal{F})$.

Proof. The Tate resolution $\mathbf{T}(\mathcal{F})$ is determined by any differential $\phi_{i}: T^{i}(\mathcal{F}) \rightarrow$ $T^{i+1}(\mathcal{F})$, because $\mathbf{T}^{\geq i+1}(\mathcal{F})$ is the minimal injective resolution of $\operatorname{im} \phi_{i}$ and $\mathbf{T}^{\leq i}(\mathcal{F})$ the minimal projective resolution of $\operatorname{im} \phi_{i}$. Moreover $\mathbf{T}(\mathcal{F})$ determines the Beilinson monad and hence $\mathcal{F}$. Thus it suffices to reconstruct one of the differentials of $\mathbf{T}(\mathcal{F})$ from $\mathbf{U}_{l}(\mathcal{F})$.

The degrees of the generators of the free module in $T^{e}(\mathcal{F})$ range (potentially) from $e-k$ to $e$. Thus the degrees of the generators of $T^{-1}(\mathcal{F})$ and $T^{0}(\mathcal{F})$ range at most from $-k-1$ to 0 . Replacing the summands $\Lambda^{p} U$ of $\mathbf{U}_{l}(\mathcal{F})$ by $E(p)$ and the maps by the corresponding matrices of exterior forms according to Proposition 1.1 (b), we recover the differential $T^{-1}(\mathcal{F}) \rightarrow T^{0}(\mathcal{F})$ of the Tate resolution.

Now we come to the case needed for the construction of the Chow divisor. If $\mathcal{B}$ is a finite complex of locally free sheaves

$$
\mathcal{B}: 0 \rightarrow \ldots \rightarrow \mathcal{B}^{j} \rightarrow \mathcal{B}^{j+1} \rightarrow \ldots \rightarrow 0
$$

then the determinant bundle of $\mathcal{B}$ is defined to be

$$
\operatorname{det}(\mathcal{B})=\prod_{j \text { even }} \operatorname{det}\left(\mathcal{B}^{j}\right) \otimes \prod_{j \text { odd }} \operatorname{det}\left(\mathcal{B}^{j}\right)^{*} .
$$

If $\mathcal{B}$ is generically exact, then there is a Cartier divisor called the determinant divisor of $\mathcal{B}$ which measures the part of the homology of $\mathcal{B}$ supported in codimension 1 ; see Knudsen and Mumford 1976] or [Gel'fand et al. 1994, Appendix A] for the general definition. If $\mathcal{F}$ is a $k$-dimensional coherent sheaf on $\mathbf{P}(W)$, then the Chow form Chow $(\mathcal{F})$ is the equation of the Chow divisor of $\mathcal{F}$. It is a section of $\mathcal{O}_{\mathbf{G}_{k+1}}(\operatorname{deg} \mathcal{F})$ 
defined up to multiplication by a scalar. The following theorem is a more explicit version the main result of [Knudsen and Mumford 1976, Chapter II].

Theorem 1.4. Let $\mathcal{F}$ be a coherent sheaf on $\mathbf{P}(W)$. If $\operatorname{dim} \mathcal{F}=k$, then the Chow divisor of $\mathcal{F}$ is the determinant divisor of the complex $\mathbf{U}_{k+1}(\mathcal{F})$. Moreover, in codimension 1 the only homology of this complex is at the 0-th term.

We give a proof for the reader's convenience:

Proof. We may assume that the ground field is algebraically closed. Since $\mathbf{U}_{k+1}(\mathcal{F})$ represents $\mathbf{R} \pi_{2 *}\left(\pi_{1}^{*} \mathcal{F}\right)$, its divisor does not pass through any point $u$ of the Grassmannian such that $\operatorname{supp}(\mathcal{F}) \cap \mathbf{P}(W / u)=\emptyset$. For a general point $u$ of a component of the zero locus of Chow $(\mathcal{F})$ the subspace $\mathbf{P}(W / u)$ meets the support of $\mathcal{F}$ in a single point which belongs to a unique component $X$ of the support. Over the residue class field $\kappa(u)$ of $u \in \mathbf{G}_{k+1}$ we have

$$
\operatorname{dim}_{\kappa(u)}\left(\pi_{2 *} \pi_{1}^{*} \mathcal{F}\right) \otimes \kappa(u)=\operatorname{dim}_{\kappa(u)} \mathrm{H}^{0}\left(\mathcal{F} \otimes \mathcal{O}_{\mathbf{P}(W / U)}\right)=\operatorname{length}\left(\mathcal{F} \otimes \mathcal{O}_{\mathbf{P}(W / u), X}\right)
$$

and the higher direct images vanish. Thus $\mathbf{R} \pi_{2 *}\left(\pi_{1}^{*} \mathcal{F}\right)$ is generically quasiisomorphic to $\pi_{2 *}\left(\pi_{1}^{*} \mathcal{F}\right)$, whose associated divisor is the Chow divisor of $\mathcal{F}$.

\section{UlRich SheAVES}

If $\mathcal{F}$ is a $k$-dimensional sheaf on $\mathbf{P}^{n}$ and $\mathbf{U}_{k+1}(\mathcal{F})$ is a two term complex, then the determinant section of $\mathbf{U}_{k+1}(\mathcal{F})$ is the determinant of a morphism between bundles. This situation corresponds to the case where the Tate resolution of $\mathcal{F}$ has "Betti diagram" of the form

$$
\begin{array}{cccccc}
h^{k} \mathcal{F}(-k-3) & h^{k} \mathcal{F}(-k-2) & h^{k} \mathcal{F}(-k-1) & h^{k} \mathcal{F}(-k) & 0 & 0 \\
0 & 0 & h^{k-1} \mathcal{F}(-k) & h^{k-1} \mathcal{F}(-k+1) & 0 & 0 \\
\vdots & \vdots & \vdots & \vdots & \vdots & \vdots \\
0 & 0 & h^{1} \mathcal{F}(-2) & h^{1} \mathcal{F}(-1) & 0 & 0 \\
0 & 0 & h^{0} \mathcal{F}(-1) & h^{0} \mathcal{F} & h^{0} \mathcal{F}(1) & h^{0} \mathcal{F}(2)
\end{array}
$$

Here, by the Betti diagram of $\mathbf{T}(\mathcal{F})$ we mean the table whose $(i, j)$ entry is the number of generators of degree $j-i$ required by the $j$-th free module $T^{i}$ in $\mathbf{T}(\mathcal{F})$; by Eisenbud et al. 2001 this is the dimension of $\mathrm{H}^{i}(\mathcal{F}(j-i)$ ). (This is almost the same as the Betti diagram in the programs Macaulay of Bayer and Stillman or Macaulay2 of Grayson and Stillman, except that we think of the arrows in the resolution as going from left to right. This change of convention is convenient because of the fact that the generators of $E$ have negative degree.) For reasons that will become clear in a moment, we will call a sheaf $\mathcal{F}$ with cohomology as above a weakly Ulrich sheaf.

An even better situation occurs when the Tate resolution has Betti diagram of the form

$$
\begin{array}{ccccccc}
\ldots & h^{k} \mathcal{F}(-k-3) & h^{k} \mathcal{F}(-k-2) & h^{k} \mathcal{F}(-k-1) & 0 & 0 & 0 \\
0 & 0 & 0 & 0 & 0 & 0 & \\
\vdots & \vdots & \vdots & \vdots & \vdots & \vdots & \\
0 & 0 & 0 & 0 & 0 & 0 & \\
0 & 0 & 0 & h^{0} \mathcal{F} & h^{0} \mathcal{F}(1) & h^{0} \mathcal{F}(2) & \ldots
\end{array}
$$

In this case we see from the previous section that the Chow form of $\mathcal{F}$ is the determinant of the $h^{0}(\mathcal{F}) \times h^{k} \mathcal{F}(-k-1)$ matrix whose entries are linear forms 
in the Plücker coordinates on the Grassmannian $\mathbf{G}_{k+1}$. (It follows that $h^{0} \mathcal{F}=$ $h^{k} \mathcal{F}(-k-1)=\operatorname{deg}(\mathcal{F})$, which one can easily see in other ways as well.)

Modules whose associated sheaf have this sort of Tate resolution were first studied in Ulrich 1984. We will call them Ulrich sheaves. Thus a $k$-dimensional sheaf $\mathcal{F}$ on $\mathbf{P}$ is an Ulrich sheaf if $\mathcal{F}$ has no intermediate cohomology - that is, $\mathrm{H}^{q}(\mathcal{F}(d))=0$ for $1 \leq q \leq k-1$ and all $d$-and $\mathrm{H}^{0}(\mathcal{F}(j))=0$ for $j<0$ while $\mathrm{H}^{k}(\mathcal{F}(j))=0$ for $j \geq-k$. Since an Ulrich sheaf has no intermediate cohomology, its restriction to the nonsingular part of $X$ is automatically a vector bundle.

We can characterize Ulrich sheaves without referring to all of the cohomology in several elementary ways. Since every 0-dimensional sheaf is an Ulrich sheaf, we will henceforward ignore this case.

Proposition 2.1. Let $\mathcal{F}$ be a coherent, $k$-dimensional sheaf on the projective space $\mathbf{P}=\mathbf{P}^{n}$ over $K$ with $k>0$. The following are equivalent:

(a) $\mathcal{F}$ is an Ulrich sheaf.

(b) $\mathrm{H}^{i} \mathcal{F}(-i)=0$ for $i>0$ and $\mathrm{H}^{i} \mathcal{F}(-i-1)=0$ for $i<k$.

(c) If the support of $\mathcal{F}$ is a scheme $X$, then for some (respectively all) finite linear projections $\pi: X \rightarrow \mathbf{P}^{k}$ the sheaf $\pi_{*} \mathcal{F}$ is the trivial sheaf $\mathcal{O}_{\mathbf{P}^{k}}^{t}$ for some t.

(d) The module $M:=\mathrm{H}_{*}^{0}(\mathcal{F}):=\bigoplus_{d} \mathrm{H}^{0}(\mathcal{F}(d))$ of twisted global sections is an Ulrich module, in the sense of [Backelin and Herzog, 1989]; that is, $M$ is a Cohen-Macaulay module of dimension $k+1$ over the homogeneous coordinate ring $S=k\left[x_{0}, \cdots, x_{n}\right]$ of $\mathbf{P}$, whose number of generators is equal to $\operatorname{deg} \mathcal{F}$, or equivalently whose $S$-free resolution

$$
\mathbf{F}: 0 \rightarrow F_{n-k} \stackrel{\varphi_{n-k}}{\longrightarrow} \ldots \stackrel{\varphi_{2}}{\longrightarrow} F_{1} \stackrel{\varphi_{1}}{\longrightarrow} F_{0} \rightarrow M \rightarrow 0
$$

is linear in the sense that $F_{i}$ is generated in degree $i$ for every $i$.

Proof. (a) $\Rightarrow$ (b) is trivial.

(b) $\Rightarrow$ (c) By the finiteness and linearity of $\pi$ we have $\mathrm{H}^{i}(\mathcal{F}(j))=\mathrm{H}^{i}\left(\left(\pi_{*}\right) \mathcal{F}(j)\right)$. The vanishing of cohomology of (b) gives vanishing for $\pi_{*} \mathcal{F}$ which characterizes the trivial vector bundles on $\mathbf{P}^{k}$.

(c) $\Rightarrow$ (d) By (c) $M=\mathrm{H}_{*}^{0}(\mathcal{F})$ is a free module over $K\left[x_{0}, \ldots, x_{k}\right]=\mathrm{H}_{*}^{0}\left(\mathcal{O}_{\mathbf{P}^{k}}\right)$ generated in degree 0 . Thus $M$ is a linear Cohen-Macaulay module, that is, an Ulrich module.

(d) $\Rightarrow$ (a) The equivalence of the two characterizations of Ulrich modules given in (d) may be found in [Brennan et al. 1987, Prop. 1.5]. The fact that a graded $S$ module $M$ is 0-regular if and only if the free resolution of $M_{\geq 0}$ is linear is proved in [Eisenbud and Goto 1984] (see also [Eisenbud 1995, Theorem 20.18]). If $M$ is a $k+$ 1-dimensional Cohen-Macaulay module with linear resolution, then the associated sheaf $\mathcal{F}$ is also 0-regular. The Cohen-Macaulay property of $M$ gives the vanishing of the intermediate cohomology of $\mathcal{F}$ and (since $\operatorname{dim} M=k+1>1$ ) also shows that $M=\mathrm{H}_{*}^{0}(\mathcal{F})$. Thus $\mathrm{H}^{0}(\mathcal{F}(j))=0$ for $j<0$, and $\mathcal{F}$ is Ulrich.

From the linearity of the resolution $\mathbf{F}$ of an Ulrich module $M$ it follows, for example, that the rank of $F_{i}$ is $\left(\begin{array}{c}n-k \\ i\end{array}\right) \cdot \operatorname{rank} F_{0}$; to see this, reduce modulo a maximal regular sequence and observe that $M$ must reduce to a direct sum of copies of the residue field $K$. In particular, $\operatorname{rank} F_{n-k}=\operatorname{rank} F_{0}$, and this rank is equal to the 
degree of $\mathcal{F}$. (For more details, see, for example, Brennan et al. 1987].) The same kind of argument gives:

Corollary 2.2. If $\mathcal{F}$ is an Ulrich sheaf of dimension $k$ on $\mathbf{P}^{r}$, then $\chi(\mathcal{F}(e))=$ $h^{0}(\mathcal{F})\left(\begin{array}{c}e+k \\ k\end{array}\right)$.

In Theorem [5.1 we will generalize this to sheaves on $X$ that are Ulrich sheaves for the $d$-uple embedding of $\mathcal{F}$.

In our applications we will be particularly interested in the case where the Ulrich sheaf is a vector bundle on its support and is self-dual up to a twist. In this case the criterion above can be simplified:

Corollary 2.3. Let $\mathcal{F}$ be a vector bundle on a $k$-dimensional Gorenstein scheme $X \subset \mathbf{P}^{r}$. If $\mathcal{F} \cong \mathcal{F}^{*}(k+1) \otimes \omega_{X}$, then $\mathcal{F}$ is an Ulrich sheaf on $\mathbf{P}^{r}$ if and only if $\mathcal{F}$ is 0 -regular.

Proof. The 0-regularity implies that $\mathrm{H}^{i}(\mathcal{F}(j))=0$ for $j>-i$. The rest of the necessary vanishing follows from Serre duality.

First examples. Brennan, Herzog and Ulrich discovered that linear determinantal varieties have rank one Ulrich modules (Brennan et al. 1987]), so we can give Bézout expressions for their Chow forms using the ideas above. This series of examples includes rational normal scrolls, Bordiga-White surfaces and many more. We can give a different description of their Ulrich modules as follows:

Example 2.4. Let $\varphi: F \rightarrow G$ with $F=\bigoplus_{1}^{f} \mathcal{O}$ and $G=\bigoplus_{1}^{g} \mathcal{O}(1), f \leq g$, be a linear $f \times g$ matrix on $\mathbf{P}^{n}$ which drops rank in expected codimension $(f-g+1)$. The Eagon-Northcott type complex

$$
0 \rightarrow \Lambda^{f} F \otimes D_{f-g+1} G^{*} \rightarrow \ldots \rightarrow \Lambda^{g} F \otimes G^{*} \rightarrow \Lambda^{g-1} F \rightarrow \mathcal{F} \rightarrow 0
$$

(see [Eisenbud 1995, Theorem A2.10]) is a linear resolution of a module annihilated by the maximal minors of $\varphi$ and has length $f-g+1$. It is thus the resolution of an Ulrich sheaf on $X=V\left(I_{g}(\varphi)\right.$ ), and one can check that the sheaf has rank 1 (it is isomorphic, in the generic case, to $I_{g-1} \varphi^{\prime}$, the ideal generated by the $g-1 \times g-1$ minors of the submatrix $\varphi^{\prime}$ obtained from $\varphi$ by omitting one row). Hence the Chow form $\operatorname{Chow}(X)=\operatorname{Chow}(\mathcal{F})$ is polynomial of degree $\left(\begin{array}{c}f \\ g-1\end{array}\right)$ in the Plücker coordinates, and $\operatorname{deg} X=\left(\begin{array}{c}f \\ g-1\end{array}\right)$.

Example 2.5. Consider the rational normal scroll $S(2,1) \subset \mathbf{P}^{4}$ defined by

$$
\varphi=\left(\begin{array}{lll}
x_{0} & x_{1} & x_{3} \\
x_{1} & x_{2} & x_{4}
\end{array}\right)
$$

Using the Ulrich sheaf $\mathcal{F}$ as above and Theorem 3.1 we obtain its Chow form as the determinant of the matrix

$$
\left(\begin{array}{ccc}
{[034]} & {[013]} & {[023]} \\
-[134] & {[023]+[014]} & -[123]-[024] \\
{[234]} & -[024] & {[124]}
\end{array}\right) .
$$

The Chow forms of rational normal scrolls have further interpretations: Consider $(r+1)$-dimensional spaces $\alpha$ of sections of bundles $\bigoplus_{i=1}^{r} \mathcal{O}_{\mathbf{P}^{1}}\left(d_{i}\right)$. The Chow form 
of the scroll $S\left(d_{1}, \ldots, d_{r}\right) \subset \mathbf{P}^{N}$ with $N+1=\sum_{i}\left(d_{i}+1\right)$ describes those $\alpha$, where the minors of the corresponding morphism

$$
\mathcal{O}_{P^{1}}^{r+1} \stackrel{\alpha}{\rightarrow} \bigoplus_{i=1}^{r} \mathcal{O}_{\mathbf{P}^{1}}\left(d_{i}\right)
$$

have a common zero. Such formulas were also worked out by Henri Lombardi and J.-P. Jouanolou (unpublished).

In the case of $S(2,1)$ there is also an interpretation for plane conics with one assigned base point: Since $S(2,1)$ is the image of $\mathbf{P}^{2}$ by the linear system of conics with a single assigned base point, say $(1: 0: 0)$, its Chow form describes those 3 -dimensional subspaces of conics which have a further base point. In Section 5 we will generalize this example to forms of any degree on $\mathbf{P}^{2}$ with several simple assigned base points.

From the point of view of examples, it is interesting to note that if two schemes in projective spaces support (weakly) Ulrich sheaves, then so does their Segre product:

Proposition 2.6. Let $\mathcal{F}_{1}$ be a coherent sheaf on $\mathbf{P}\left(W_{1}\right)$ and let $\mathcal{F}_{2}$ be a coherent sheaf on $\mathbf{P}\left(W_{2}\right)$. Set $d=\operatorname{dim}\left(\mathcal{F}_{1}\right)$. Let $\mathcal{G}$ be the Segre product of $\mathcal{F}_{1}$ with $\mathcal{F}_{2}(d)$ on $\mathbf{P}=\mathbf{P}\left(W_{1} \otimes W_{2}\right)$; that is, $\mathcal{G}=\left(\pi_{1}^{*} \mathcal{F}_{1}\right) \otimes\left(\pi_{2}^{*} \mathcal{F}_{2}(d)\right)$ on the Segre variety $\mathbf{P}\left(W_{1}\right) \times$ $\mathbf{P}\left(W_{2}\right) \subset \mathbf{P}$.

(a) If $\mathcal{F}_{1}, \mathcal{F}_{2}$ are weakly Ulrich, then $\mathcal{G}$ is weakly Ulrich.

(b) If $\mathcal{F}_{1}, \mathcal{F}_{2}$ are Ulrich, then $\mathcal{G}$ is Ulrich.

Of course a similar result holds for the Segre product of $\mathcal{F}_{1}\left(\operatorname{dim} \mathcal{F}_{2}\right)$ and $\mathcal{F}_{2}$.

Proof. Both parts follow easily from the Künneth formula

$$
\mathrm{H}^{i}(\mathcal{G}(m))=\bigoplus_{i=j+k} \mathrm{H}^{j}\left(\mathcal{F}_{1}(m)\right) \otimes \mathrm{H}^{k}\left(\mathcal{F}_{2}(d+m)\right)
$$

For example, in part (a) we need $\mathrm{H}^{j}\left(\mathcal{F}_{1}(-j-k-2)\right) \otimes \mathrm{H}^{k}\left(\mathcal{F}_{2}(d-j-k-2)\right)=0$ when $j+k<d+\operatorname{dim} \mathcal{F}_{2}$. If $j<d$, then the first factor vanishes since $\mathcal{F}_{1}$ is weakly Ulrich, while if $j=d$, then the second factor vanishes for the same reason.

Corollary 2.7. With notation as in Proposition [2.6, suppose that $\mathcal{F}_{1}, \mathcal{F}_{2}$ are Ulrich sheaves of dimensions $d_{1}, d_{2}$, and let $E_{\text {Segre }}=\wedge\left(\left(W_{1} \otimes W_{2}\right)^{*}\right)=\wedge\left(W_{1}^{*} \otimes W_{2}^{*}\right)$. The map

$$
\mathrm{H}^{d_{1}+d_{2}}\left(\mathcal{G}\left(-d_{1}-d_{2}-1\right)\right) \otimes \omega_{E_{\text {Segre }}} \rightarrow \mathrm{H}^{0}(\mathcal{G}) \otimes \omega_{E_{\text {Segre }}}
$$

in the Tate resolution of $\mathcal{G}$ is derived from the tensor product of the corresponding maps for $\mathcal{F}_{1}$ and $\mathcal{F}_{2}$ over $\bigwedge W_{1}^{*}$ and $\bigwedge W_{2}^{*}$, respectively, via the canonical injection $\bigwedge W_{1}^{*} \otimes \wedge W_{2}^{*} \subset \wedge\left(W_{1}^{*} \otimes W_{2}^{*}\right)$.

It follows that in situations where we can compute a Bézout expression for the Chow forms of $\mathcal{F}_{1}$ and $\mathcal{F}_{2}$, we can also compute a Bézout expression for the Chow form of the Segre product. Similar remarks and formulas hold in the case of weakly Ulrich sheaves and Stiefel expressions of the Chow form. 


\section{Chow Forms as Determinants And Pfaffians}

Throughout this section we will work with a sheaf $\mathcal{F}$ of dimension $k$ on $\mathbf{P}^{n}=$ $\mathbf{P}(W)$. For simplicity, we will write $\mathbf{U}$ for the functor $\mathbf{U}_{k+1}$ defined in Section 1 We set $c=n-k$, the codimension of $\mathcal{F}$. Let

$$
\mathbf{T}(\mathcal{F}): \cdots \rightarrow T^{-1} \stackrel{\varphi_{\mathcal{F}}}{\longrightarrow} T^{0} \rightarrow \cdots
$$

be the Tate resolution of $\mathcal{F}$, with "middle" map $\varphi_{\mathcal{F}}$. We have seen in the previous section that if $\mathcal{F}$ is weakly Ulrich, then the complex $\mathbf{U}(\mathcal{F})$ is given by a single map $\Psi_{\mathcal{F}}: \mathbf{U}\left(\varphi_{\mathcal{F}}\right)$ of vector bundles on the Grassmannian, and the Chow form of $\mathcal{F}$ is the determinant of $\Psi_{\mathcal{F}}$.

One way to compute $\varphi_{\mathcal{F}}$ is as the $m$-th syzygy matrix over the exterior algebra starting from the multiplication tensor

$$
\mathrm{H}^{0}(\mathcal{F}(m-1)) \rightarrow \mathrm{H}^{0}(\mathcal{F}(m)) \otimes\left(\mathrm{H}^{0}\left(\mathcal{O}_{\mathbf{P}(W)}(1)\right)\right)^{*}
$$

for some $m \geq 2$. In this section we describe another method which, in the Ulrich case, makes $\varphi_{\mathcal{F}}$ explicit in terms of the minimal free resolution of $\mathcal{F}$. The tools we develop will allow us to show that if $\mathcal{F}$ is skew-symmetrically self-dual in a natural sense, then the complex $\mathbf{U}(\mathcal{F})$ is skew-symmetric, and in particular $\Psi_{\mathcal{F}}$ is skew-symmetric. When $\mathcal{F}$ is also weakly Ulrich, the square root of the Chow form of $\mathcal{F}$ is the Pfaffian of $\Psi_{\mathcal{F}}$. In particular, when $\mathcal{F}$ is in addition a sheaf of rank 2 supported on a variety $X$, the Chow form of $X$ itself is the Pfaffian of $\Psi_{\mathcal{F}}$.

We first review the basics of the Bernstein-Gel'fand-Gel'fand correspondence, Bernstein, Gel'fand and Gel'fand 1978 in the style of Eisenbud et al. 2001. Let $V=\operatorname{Hom}_{K}(W, K)$ be the dual vector space and let $E=\bigwedge V$ be its exterior algebra, graded with $V$ in degree -1 as usual. There is a functor $\mathbf{L}$ from graded $E$-modules to linear free complexes over $S$ defined as follows: If $P$ is a graded $E$-module, then $\mathbf{L}(P)$ is the complex

$$
L: \quad \cdots \rightarrow S \otimes_{K} P_{i-1} \stackrel{\alpha}{\longrightarrow} S \otimes_{K} P_{i} \stackrel{\alpha}{\rightarrow} S \otimes_{K} P_{i+1} \rightarrow \cdots
$$

such that $\alpha_{i}(1 \otimes p)=\sum x_{i} \otimes e_{i} p$, where $\left\{x_{i}\right\}$ and $\left\{e_{i}\right\}$ are dual bases of $W$ and $V$. Every linear free complex $L$ over $S$ can be written as $L=\mathbf{L}(P)$ for a unique graded $E$-module $P$.

It is easy to write down the module structure of $P$ from the differentials of $L$. Giving a multiplication map $V \otimes P_{i} \rightarrow P_{i-1}$ is equivalent to giving the "adjoint" map $P_{i} \rightarrow P_{i-1} \otimes W$. The (linear) differential $L_{i}=S \otimes P_{i} \rightarrow L_{i-1}=S \otimes P_{i-1}$ of $L$ is defined by the desired map $P_{i} \rightarrow W \otimes P_{i-1}=P_{i-1} \otimes W$. The skew-commutativity and associativity necessary for an $E$-module structure follow immediately from the fact that $L$ is a complex.

We will use this construction to write down the middle map $\varphi_{\mathcal{F}}$ of the Tate resolution of an arbitrary sheaf. When $\mathcal{F}$ is Ulrich, our result takes the form given in Theorem 0.3 which allows the computation of the Chow form directly from the free resolution of the Ulrich module of twisted global sections of $\mathcal{F}$. But in the general case we must replace the resolution by a linear free monad, defined in Eisenbud et al. 2001, Example 8.5 and Proposition 8.6].

Theorem 3.1. Let $\mathcal{F}$ be a coherent sheaf on $\mathbf{P}^{n}$. There is a unique linear free complex of $S$-modules

$$
L=\mathbf{L}(\mathcal{F}): \quad \cdots \stackrel{\alpha_{-2}}{\longrightarrow} L^{-1} \stackrel{\alpha_{-1}}{\longrightarrow} L^{0} \stackrel{\alpha_{0}}{\longrightarrow} L^{1} \stackrel{\alpha_{1}}{\longrightarrow} \cdots,
$$


called the linear free monad of $\mathcal{F}$, such that $L^{-i}=S(-i) \otimes P_{i}$ and $L^{i}=0$ if $|i|>n$, with the property that the sheafification of the homology of $L$ is zero except for $\widetilde{\mathrm{H}^{0}(L)}=\mathcal{F}$. The complex $\mathbf{L}(\mathcal{F})$ is functorial in $\mathcal{F}$ and may be constructed explicitly by means of the Bernstein-Gel'fand-Gel'fand correspondence: Setting $P=\operatorname{im} \varphi_{\mathcal{F}}$ : $T^{-1} \rightarrow T_{0}$, the image of the middle map of the Tate resolution of $\mathcal{F}$, we have $\mathbf{L}(\mathcal{F})=\mathbf{L}(P)$.

In the case when the module of twisted global sections $M=\bigoplus_{d} \mathrm{H}^{0}(\mathcal{F}(d))$ of $\mathcal{F}$ has a linear free resolution - for example, when $\mathcal{F}$ is Ulrich - the uniqueness statement shows that this resolution is the linear free monad $\mathbf{L}$, and this gives us an alternate method of constructing $\mathbf{L}$. It would be interesting to have such an alternate method in general.

Associated to $L=\mathbf{L}(\mathcal{F})=\mathbf{L}(P)$ are maps

$$
\varphi_{-i,-j}: \quad P_{i} \rightarrow \bigwedge^{i-j} W \otimes P_{j}
$$

adjoint to the multiplication maps $\bigwedge^{i-j} V \otimes P_{i} \rightarrow P_{i-j}$ that define the $E$-module structure on $P$. These may also be computed directly from the differentials of $L$, as follows: Since the differentials of $L$ are given by matrices of linear forms, they are determined by vector space maps $P_{i} \rightarrow W \otimes P_{i-1}$. Composing $j$ such maps, we get $P_{i} \rightarrow \bigotimes_{1}^{j} W \otimes P_{i-j}$. The image of this map is actually contained in $\bigwedge^{j} W \otimes P_{i-j}$ because the composition of the original differentials is zero over the symmetric algebra. The map $\varphi_{-i,-j}$ is the induced map $P_{i} \rightarrow \bigwedge^{j} W \otimes P_{i-j}$. In characteristic 0 , it could also be obtained by composing matrices representing the differentials as if they were matrices over the exterior algebra and dividing by $j$ !.

We can now describe the map $\varphi_{\mathcal{F}}: T^{-1}(\mathcal{F}) \rightarrow T^{0}(\mathcal{F})$. Let $(V)$ be the ideal of elements of negative degree in $E$ (the augmentation ideal) and define graded vector spaces $A$ and $B$ by

$$
A=P /(V) P, \quad B=\{p \in P \mid(V) p=0\} .
$$

The map $T^{-1}(\mathcal{F}) \rightarrow P$ is a projective cover: that is, a minimal map from a free $E$-module onto $P$. It follows from Nakayama's Lemma that we may make the identification $T^{-1}(\mathcal{F})=E \otimes A$, and the map to $T^{-1} \rightarrow P$ is determined by the data of a splitting $\eta: A \rightarrow P$ (as graded vector spaces) of the natural projection map $P \rightarrow A$.

Recall that the injective envelope of the residue field $K=E /(V)$ may be described canonically as $\omega_{E}=E \otimes \bigwedge^{n+1} W$, whose degree $i$ component is $\bigwedge^{n+1-i} V=$ $\bigwedge^{i} W$. The map $P \rightarrow T^{0}(\mathcal{F})$ is an injective envelope. Dually to the situation for projective covers, an injective envelope $P \rightarrow G$ is uniquely determined by a splitting $\pi: P \rightarrow B$ of the inclusion $B \subset P$; we take $T^{0}(\mathcal{F})=\omega_{E} \otimes B$, and the map $P \rightarrow T^{0}(\mathcal{F})$ is the unique map to $\omega_{E} \otimes B$ whose composition with the projection to $\left(\omega_{E}\right)_{0} \otimes B=B$ is $\pi$.

By Eisenbud et al. 2001 Theorem 4.1], the components of $A$ and $B$ are

$$
A_{c+i}=\mathrm{H}^{k-i}(\mathcal{F}(i-k-1)), \quad B_{j}=\mathrm{H}^{j}(\mathcal{F}(-j)),
$$

where as usual $c$ denotes the codimension $n-k$ of $\mathcal{F}$. This identification will be useful later. 
Summarizing:

Theorem 3.2. If $\mathcal{F}$ is a coherent sheaf on $\mathbf{P}^{n}$ and $L=\mathbf{L}(\mathcal{F})=\mathbf{L}(P)$ is its linear monad, then, with notation as above, the middle map

$$
\varphi_{\mathcal{F}}: E \otimes A=T^{-1}(\mathcal{F}) \rightarrow T^{0}(\mathcal{F})=\omega_{E} \otimes B
$$

in the Tate resolution of $\mathcal{F}$ has components

$$
\begin{aligned}
\mathrm{H}^{k-i+c} & (\mathcal{F}(i-c-k-1)) \\
= & A_{i} \stackrel{\eta_{i}}{\longrightarrow} P_{i} \stackrel{\varphi_{-i,-j}}{\longrightarrow} \bigwedge^{i-j} W \otimes P_{j} \stackrel{\pi_{j}}{\longrightarrow} \bigwedge^{i-j} W \otimes B_{j} \\
= & \bigwedge^{i-j} W \otimes \mathrm{H}^{j}(\mathcal{F}(-j)) .
\end{aligned}
$$

For example, if $L$ is a free resolution of an Ulrich sheaf, by Eisenbud et al. 2001. Proposition 8.7] $A=P_{c}$ and $B=P_{0}$, so in that case $\varphi_{\mathcal{F}}$ is the map induced by the map $\varphi_{-c, 0}$, and the map $\Psi_{\mathcal{F}}$ is the one given in Theorem 0.3 . No choice of $\eta$ and $\pi$ is involved because $A=P_{-c}, B=P_{0}$ in that case.

3.1. The skew-symmetry of $\mathbf{U}(\mathcal{F})$. We now show that appropriate symmetry or skew-symmetry of $\mathcal{F}$ makes $\mathbf{U}(\mathcal{F})$ symmetric or skew-symmetric. The functor

$$
D: \mathcal{F} \mapsto \mathcal{E} x t^{c}\left(\mathcal{F}, \omega_{\mathbf{P}^{n}}\right)(k+1)
$$

defines a duality on the category of $k$-dimensional Cohen-Macaulay sheaves on $\mathbf{P}^{n}$ and there is a canonical morphism $\iota: \mathcal{F} \rightarrow D D(\mathcal{F})$. Let $\epsilon= \pm 1$. As with any duality, we say that a morphism $\sigma: \mathcal{F} \rightarrow D(\mathcal{F})$ is $\epsilon$-symmetric if

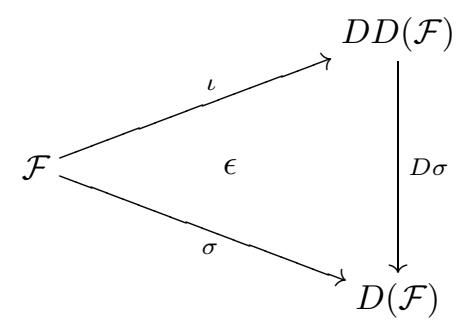

commutes up to the $\operatorname{sign} \epsilon$. In case $\epsilon=1$, we say that $\mathcal{F}$ is symmetric; if $\epsilon=-1$, then $\mathcal{F}$ is called skew-symmetric.

Theorem 3.3. Suppose that $\mathcal{F}$ is a Cohen-Macaulay sheaf of dimension $k$ on $\mathbf{P}^{n}$. Any $\epsilon$-symmetric isomorphism $\mathcal{F} \rightarrow D(\mathcal{F})$ induces an $\epsilon$-symmetric isomorphism

$$
\mathbf{U}(\mathcal{F}) \rightarrow \mathcal{H o m}_{\mathbf{G}_{k+1}}\left(\mathbf{U}(\mathcal{F}), \mathcal{O}_{\mathbf{G}_{k+1}}(-1)\right)[1]
$$

In particular the map $\mathbf{U} T^{-1}(\mathcal{F}) \stackrel{\Psi_{\mathcal{F}}}{\longrightarrow} \mathbf{U} T^{0}(\mathcal{F})$ is $\epsilon$-symmetric, and for $j>1$ the map $\mathbf{U} T^{-j}(\mathcal{F}) \rightarrow \mathbf{U} T^{-j+1}(\mathcal{F})$ is dual to $\mathbf{U} T^{j-1}(\mathcal{F}) \rightarrow \mathbf{U} T^{j}(\mathcal{F})$.

We postpone the proof to state a corollary and a lemma.

If $\mathcal{F}$ is skew-symmetric, we define the Pfaffian of the skew-symmetric complex $\mathbf{U}(\mathcal{F})$ by taking an appropriate Pfaffian of the middle map $\mathbf{U} T^{-1}(\mathcal{F}) \stackrel{\Psi_{\mathcal{F}}}{\longrightarrow} \mathbf{U} T^{0}(\mathcal{F})$ times the alternating product of those terms from the determinant of $\mathbf{U}(\mathcal{F})$ that are associated with the maps $\mathbf{U} T^{-j}(\mathcal{F}) \rightarrow \mathbf{U} T^{-j+1}(\mathcal{F})$ for $j>0$. The determinant of $\mathbf{U}(\mathcal{F})$ is then the square of the Pfaffian of $\mathbf{U}(\mathcal{F})$. 
Corollary 3.4. Assume that the characteristic of the ground field is not 2 . If $\mathcal{F}$ is a skew-symmetric Cohen-Macaulay sheaf of rank 2 on a $k$-dimensional subscheme $X \subset \mathbf{P}^{n}$ such that $\bigwedge^{2} \mathcal{F} \cong \omega_{X}(k+1)$, then the Chow form of $X$ is the Pfaffian of the complex $\mathbf{U}(\mathcal{F})$. In particular if $\mathcal{F}$ is weakly Ulrich, then the Chow form of $X$ is the Pfaffian of the skew-symmetric map of vector bundles $\mathbf{U}\left(\Psi_{\mathcal{F}}\right)$.

Remark. In order to include the case of characteristic 2, we would have to add the condition that the duality $D$ is alternating, not just skew-symmetric, and then prove the corresponding result for $\Psi_{\mathcal{F}}$. We leave this task to the interested reader.

Proof of Corollary 3.4 The skew-symmetric pairing $\mathcal{F} \otimes \mathcal{F} \rightarrow \bigwedge^{2} \mathcal{F} \cong \omega_{X}(k+1)$ gives rise to a skew-symmetric isomorphism

$$
\mathcal{F} \rightarrow \mathcal{H o m}\left(\mathcal{F}, \omega_{X}(k+1)\right) \cong \mathcal{E} x t^{c}\left(\mathcal{F}, \omega_{\mathbf{P}^{n}}\right) \cong D(\mathcal{F}) .
$$

The rest follows from Theorem 3.3 and the discussion above.

To prove Theorem 3.3 we will first analyse the map on linear monads induced by the (skew) symmetric isomorphism $\mathcal{F} \rightarrow D \mathcal{F}$. From this analysis will come a certain symmetry property of $\varphi_{\mathcal{F}}$. The map $\varphi_{\mathcal{F}}$ may be represented by a matrix of elements of $\bigwedge W$. An element $\alpha \in \bigwedge^{t} W$ induces for any integer $s$ a map (which we again call $\alpha$ ) defined by

$$
\bigwedge^{s} U \otimes \bigwedge^{v} V \stackrel{\alpha}{\rightarrow} \bigwedge^{s+t-v} U \quad: \quad u \otimes e \mapsto \alpha(e)(u)
$$

where $\alpha(e) \in \bigwedge^{v-t} V$ acts on $U$ as described in Section 1. The map $\Psi_{\mathcal{F}}$ is constructed from these pieces, so we will derive a symmetry property for $\Psi_{F}$.

The difficulty in proving Theorem 3.3 comes from the delicacy of the signs involved. For example, consider the case where the map $\varphi_{\mathcal{F}}: E(k+1-i) \rightarrow \omega_{E}(i)$ in Theorem 3.2 is given by a $1 \times 1$ matrix whose entry is in $\bigwedge^{c+2 i} W$. One might suppose that any $1 \times 1$ matrix would be symmetric and would correspond to a symmetric map of vector bundles $\Psi:\left(\bigwedge^{i} U\right)^{*} \cong \bigwedge^{k+1-i} U \otimes \bigwedge^{v} V \rightarrow \bigwedge^{i} U$ on the Grassmannian. But actually $\Psi$ is symmetric if $i$ is even and skew-symmetric if $i$ is odd. The general result we need is the following:

Lemma 3.5. Set $c=v-k-1$ and let $\alpha \in \bigwedge^{c+i+j} W$. The dual into $\mathcal{O}_{\mathbf{G}_{k+1}}(-1)$ of the map

$$
\bigwedge^{k+1-i} U \otimes \bigwedge^{v} V \stackrel{\alpha}{\rightarrow} \bigwedge^{j} U
$$

is the map

$$
\bigwedge^{k+1-j} U \otimes \bigwedge^{v} V \stackrel{(-1)^{k(i+j)+i j} \cdot \alpha}{\longrightarrow} \bigwedge^{i} U
$$

Proof of Lemma 3.5. We identify $\bigwedge^{i} U$ with $\operatorname{Hom}\left(\bigwedge^{k+1-i} U \otimes \bigwedge^{v} V, \mathcal{O}_{\mathbf{G}_{k+1}}(-1)\right)$ via the map $\tau$ sending $\beta \otimes e \in \bigwedge^{k+1-i} U \otimes \bigwedge^{v} V$ to the functional

$$
\tau: \bigwedge^{i} U \ni \chi \mapsto(\chi \wedge \beta)(e) \in \mathcal{O}_{\mathbf{G}_{k+1}}(-1) .
$$


We must show that the diagram

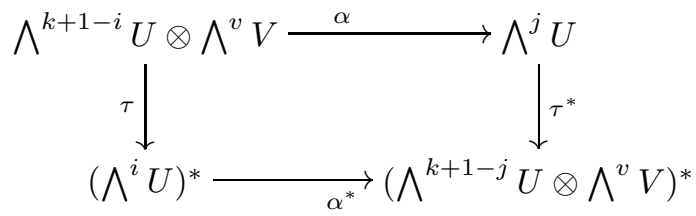

commutes up to a sign of $(-1)^{k(i+j)+i j}$. Although this is a diagram of vector bundles, we may reduce the problem to one of vector spaces by working fiberwise. For each $p \in \mathbf{G}_{k+1}$ the fiber $U_{p}$ of $U$ is a subspace of $W$, and the action of $V$ on $U_{p}$ is induced by its action on $W$. Thus the annihilator of $U_{p}$ in $V$ acts as zero on $U_{p}$, and we may therefore replace $W$ by $U_{p}$ and $V$ by $U_{p}^{*}$ and assume that $U=W$ so that $v=k+1$ and $c=0$.

From the definitions we see that

$$
\begin{array}{ll}
\alpha^{*} \tau(\beta \otimes e): & \gamma \otimes e \mapsto[((\alpha(e))(\gamma)) \wedge \beta](e), \\
\tau^{*} \alpha(\beta \otimes e): & \gamma \otimes e \mapsto[((\alpha(e))(\beta)) \wedge \gamma](e) .
\end{array}
$$

Since these expressions are multilinear in $\alpha, \beta, \gamma$, it suffices to check the case where $\alpha, \beta, \gamma$ are products of elements in some fixed basis $\left\{x_{1}, \ldots, x_{v}\right\}$ of $W$. Set $a=$ $\alpha(e) \in \bigwedge^{k+1-i-j} V$. The expressions are both zero unless $a(\beta) \wedge \gamma$ is a scalar times the product of all the basis elements $\left\{x_{1}, \ldots, x_{v}\right\}$. Under this assumption, what we are trying to prove is equivalent to the statement that

$$
a(\gamma) \wedge \beta=(-1)^{k(i+j)+i j} a(\beta) \wedge \gamma .
$$

Let $\bar{\alpha}$ be the element of $\bigwedge^{k+1-i-j} W$ such that $\alpha(e)(\bar{\alpha})=a(\bar{\alpha})=1$. Our assumptions imply that we can factorize $\gamma$ and $\beta$ as $\gamma=\gamma^{\prime} \bar{\alpha}$ and $\beta=\bar{\alpha} \beta^{\prime}$. With this notation

$$
\begin{aligned}
& a(\gamma) \wedge \beta=a\left(\gamma^{\prime} \wedge \bar{\alpha}\right) \wedge \beta=(-1)^{\gamma^{\prime} a} \gamma^{\prime} \wedge \beta=(-1)^{\gamma^{\prime} a} \gamma^{\prime} \wedge \bar{\alpha} \wedge \beta^{\prime} \\
& a(\beta) \wedge \gamma=a\left(\bar{\alpha} \wedge \beta^{\prime}\right) \wedge \gamma=\beta^{\prime} \wedge \gamma=\beta^{\prime} \wedge \gamma^{\prime} \wedge \bar{\alpha}=(-1)^{\left(\gamma^{\prime}+a\right) \beta^{\prime}} \gamma^{\prime} \wedge \bar{\alpha} \wedge \beta^{\prime},
\end{aligned}
$$

where we also write $\gamma^{\prime}, a$, and $\beta^{\prime}$ for the degrees of $\gamma^{\prime}, a$, and $\beta^{\prime}$. Thus the diagram commutes up to the sign $(-1)^{\left(\gamma^{\prime}+a\right) \beta^{\prime}+\gamma^{\prime} a}$. But $\left(\gamma^{\prime}+a\right) \beta^{\prime}+\gamma^{\prime} a=\gamma(\beta-a)+(\gamma-a) a=$ $\gamma \beta-a^{2}=(k+1-i)(k+1-j)+(k+1-i-j)^{2}$ and this is congruent modulo 2 to $k(i+j)+i j$ as required.

Proof of Theorem 3.3. We will show that the "middle" differential

$$
\mathbf{U} T^{-1}(\mathcal{F}) \stackrel{\Psi_{\mathcal{F}}}{\longrightarrow} \mathbf{U} T^{0}(\mathcal{F})
$$

is $\epsilon$ symmetric. This condition depends on an identification of $\mathbf{U} T^{0}(\mathcal{F})$ with the dual of $\mathbf{U} T^{-1}(\mathcal{F})$. Changing this identification is the same as multiplying $\Psi_{\mathcal{F}}$ by an automorphism of its source or target, so it suffices to show that $\Psi_{\mathcal{F}}$ times such an isomorphism is $\epsilon$ symmetric.

Once we know that the middle differential is $\epsilon$ symmetric, we can take the injective resolution of $P$, from which the positively indexed maps of $\mathbf{U}(\mathcal{F})$ are made, to be dual to the free resolution of $\mathbf{P}$ from which the negatively indexed maps of $\mathbf{U}(\mathcal{F})$ are made. 
To analyze $\Psi_{\mathcal{F}}$ we will make use of the analysis of $\varphi_{\mathcal{F}}$ in Theorem 3.2 We have decompositions

$$
\begin{aligned}
T^{-1}(\mathcal{F}) & =\sum_{i} A_{c+i} \otimes E(-c-i) \quad \text { and } \\
T^{0}(\mathcal{F}) & =\sum_{j} B_{-j} \otimes \omega_{E}(j) .
\end{aligned}
$$

In terms of this decomposition, the $(i, j)$ component of $\varphi_{\mathcal{F}}$ is $\pi_{j} \varphi_{-c-i, j} \eta_{-c-i}$. Applying the functor $\mathbf{U}$, we see that $\Psi_{\mathcal{F}}$ decomposes into maps

$$
\begin{aligned}
\mathbf{U}\left(A_{c+i} \otimes E(-c-i)\right) & =\mathbf{U}\left(A_{c+i} \otimes \bigwedge^{v} \otimes \omega_{E}(k+1-i)\right)=A_{c+i} \otimes \bigwedge^{v} V \otimes \bigwedge^{k+1-i} U \\
\stackrel{\left(\Psi_{\mathcal{F}}\right)_{i, j}}{\longrightarrow} \mathbf{U}\left(B_{-j} \otimes \omega_{E}(j)\right) & =B_{-j} \otimes \bigwedge^{j} U
\end{aligned}
$$

where $U$ denotes the tautological subbundle on the Grassmanian. With this indexing, we will show that the maps $\left(\Psi_{\mathcal{F}}\right)_{i, j}$ and $\left(\Psi_{\mathcal{F}}\right)_{j, i}$ are dual up to a certain sign.

As already noted, we may identify $A_{c+i}$ with $\mathrm{H}^{k-i}(\mathcal{F}(i-k-1))$ and $B_{j}$ with $\mathrm{H}^{j}(\mathcal{F}(-j))$. As we have assumed that $\mathcal{F} \cong \mathcal{E} x t^{c}\left(\mathcal{F}, \omega_{\mathbf{P}^{n}}(k+1)\right)$, we have

$$
B_{j}^{*}=\mathrm{H}^{j}(\mathcal{F}(-j))^{*}=\mathrm{H}^{j}\left(\mathcal{E} x t^{c}\left(\mathcal{F}, \omega_{\mathbf{P}^{n}}(k+1)\right)(-j)\right)^{*}=\mathrm{H}^{n-j}(\mathcal{F}(k+1-j))=A_{j}
$$

by Serre duality. With this identification it suffices to check the signs in the maps $\varphi_{\mathcal{F}}$ rather than in the maps $\varphi_{\bullet}, \bullet$.

The linear complex $\mathcal{H o m}\left(L, \omega_{\mathbf{P}^{n}}\right)(k+1)[c]$, is a linear free monad for the dual sheaf $D(\mathcal{F}) \cong \mathcal{F}$. By the uniqueness and functoriality of linear monads (Theorem 3.1), the isomorphism $\sigma$ induces an isomorphism $L \cong \mathcal{H} \operatorname{om}\left(L, \omega_{\mathbf{P}^{n}}\right)(k+1)[c]$.

To simplify notation, we set $\check{L}^{i}=D\left(L^{i}\right)=\mathcal{H o m}\left(L^{i}, \omega_{\mathbf{P}^{n}}\right)(k+1)$. We follow standard sign conventions (see, for example, Iverson 1986]) and define the dual complex $\check{L}=\mathcal{H} \operatorname{Hom}\left(L, \omega_{\mathbf{P}^{n}}\right)(k+1)$ to have differentials $(-1)^{i} \check{\alpha}_{i}$. Shifting the complex $c$ steps also introduces the $\operatorname{sign}(-1)^{c}$. Thus the isomorphism $L \rightarrow \check{L}[c]$ consists of a sequence of isomorphisms $\sigma_{j}: L^{j} \rightarrow \check{L}^{-c-j}$ as in the following diagram:

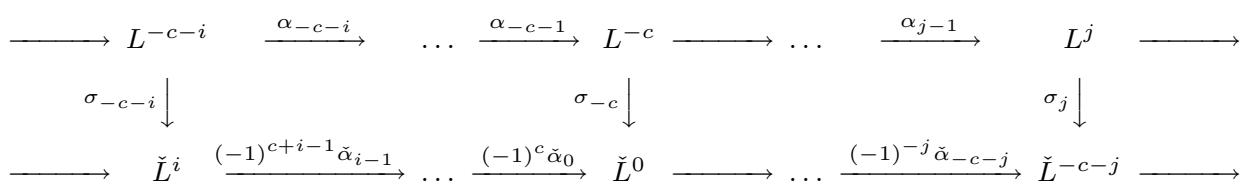

From the diagram we see that

$$
\begin{aligned}
\varphi_{-c-i, j} & =\sigma_{j} \alpha_{j-1} \otimes \ldots \otimes \alpha_{-c-i} \\
& =(-1)^{s} \check{\alpha}_{-c-j} \otimes \ldots \otimes \check{\alpha}_{i-1} \sigma_{-c-i}
\end{aligned}
$$

with $s=c(c+i+j)+\left(\begin{array}{c}c+j+1 \\ 2\end{array}\right)+\left(\begin{array}{l}i \\ 2\end{array}\right)$, where the $c(c+i+j)$ comes from the shift, and the rest is the contribution of the signs in the duality, separating the parts with positive and negative indices.

We next prove that the map $\sigma_{-c-i}$ is, up to a sign we shall identify, the dual of $\sigma_{i}$. By the uniqueness and functoriality of linear monads and the $\epsilon$ symmetry of $\sigma$, the induced map of complexes $\sigma^{\prime}: L \rightarrow \check{L}[c]$ factors as the composite $\sigma^{\prime}=\epsilon D\left(\sigma^{\prime}\right) \iota^{\prime}$ where $\iota^{\prime}$ is the canonical morphism of complexes

$$
\iota^{\prime}: L \rightarrow \mathcal{H o m}\left(\mathcal{H} \operatorname{Hom}\left(L, \omega_{\mathbf{P}^{n}}\right)[c], \omega_{\mathbf{P}^{n}}\right)[c]
$$


The components of $\iota^{\prime}$ are given by

$$
\iota_{\ell}^{\prime}: L_{\ell} \stackrel{(-1)^{(c+1)(c+\ell)} \iota}{\longrightarrow} \check{L}_{\ell}
$$

where $\iota$ denotes the canonical morphism $M \rightarrow \check{M}$ of sheaves (see also Iverson 1986 p. 73]). Thus $\sigma_{-c-i}=\epsilon(-1)^{(c+1) i} \check{\sigma}_{i} \iota$.

Combining this equation with $(*)$, we get

$$
\begin{aligned}
\varphi_{-c-i, j} & =\epsilon(-1)^{s+(c+1) i} \check{\alpha}_{-c-j} \otimes \ldots \otimes \check{\alpha}_{i-1} \check{\sigma}_{i \iota} \\
& =\epsilon(-1)^{s+t} \operatorname{transpose}\left(\sigma_{i} \alpha_{i-1} \otimes \ldots \otimes \alpha_{-c-j}\right)
\end{aligned}
$$

with $t=(c+1) i+\left(\begin{array}{c}c+i+j \\ 2\end{array}\right)$ since in the transpose matrix the tensor factors occur in the opposite order, and this tensor lies in $\bigwedge^{c+i+j} W$.

Now

$$
\begin{aligned}
s+t= & c(c+i+j)+\left(\begin{array}{c}
c+j+1 \\
2
\end{array}\right)+\left(\begin{array}{l}
i \\
2
\end{array}\right)+(c+1) i+\left(\begin{array}{c}
c+i+j \\
2
\end{array}\right) \\
= & c^{2}+c(i+j)+\frac{(c+j)^{2}+(c+j)}{2}+\frac{i^{2}-i}{2}+c i+i \\
& +\frac{(c+i+j)^{2}-(c+i+j)}{2} \\
\equiv & (c+1)(i+j)+i j \bmod 2 .
\end{aligned}
$$

By Lemma 3.5, we see that all the diagonal blocks $\left(\Psi_{\mathcal{F}}\right)_{i, i}=\mathbf{U}\left(\varphi_{-c-i, i}\right)$ will be $\epsilon$ symmetric. Because $(c+1)(i+j)+i j+k(i+j)+i j \equiv v(i+j)$, we can multiply the block matrix $\Psi_{\mathcal{F}}$ by the diagonal matrix of signs $\Delta=\bigoplus_{j}(-1)^{v j} I d_{A_{j}}$, where $I d_{A_{j}}$ is the identity map on $A_{j}$, to get a map which is $\epsilon$ symmetric; that is, setting $\Psi_{\mathcal{F}}^{\prime}=\Psi_{\mathcal{F}} \Delta$, we will have $\epsilon \mathcal{H}$ om $\left(\left(\Psi_{\mathcal{F}}^{\prime}\right), \mathcal{O}_{\mathrm{G}}(-1)\right)=\Psi_{\mathcal{F}}^{\prime}$.

\section{Curves}

The projective line. Binary forms were the starting point for the theory of resultants (Leibniz 1693, Bézout 1779] Sylvester 1840-1842]; see [Kline 1972] for some historical remarks), and they correspond to the simplest cases of Chow forms of curves. We now explain how they fit into our theory by redoing the most classical result in our language.

Example 4.1 (Binary forms). Consider the rational normal curve $\iota: \mathbf{P}^{1} \hookrightarrow$ $\mathbf{P}^{d},(s: t) \mapsto\left(s^{d}, s^{d-1} t, \ldots, t^{d}\right)$. We use $[i, j]$ for the $i j$-th Plücker coordinate of $\mathbf{G}_{2}=\mathbf{G}\left(2, \mathrm{H}^{0}\left(\mathbf{P}^{d}, \mathcal{O}(1)\right)\right)$ with respect to the given basis.

Proposition 4.2. The Chow form of the rational normal curve of degree $d$ is the determinant of the $d \times d$ symmetric matrix $A=\left(a_{i j}\right)$ with

$$
a_{i j}=\sum_{\substack{p<\min (i, j) \\ p+q=i+j-1}}[p, q]
$$

Since the rational normal curve is a linear determinantal variety, this formula could be deduced from Theorem 3.1. But from our point of view the most direct method is the computation of a map in a Tate resolution. 
Proof. Let $\mathcal{L}$ be the line bundle of degree -1 on $\mathbf{P}^{1}$. Let $\iota: \mathbf{P}^{1} \rightarrow \mathbf{P}^{d}$ be the $d$-uple embedding. The Tate resolution of $\mathcal{F}=\left(\iota_{*} \mathcal{L}\right)(1)$, the line bundle associated to the divisor of degree $d-1$, has Betti diagram

$$
\begin{array}{cccccc}
3 d & 2 d & d & - & - & - \\
- & - & - & d & 2 d & 3 d
\end{array}
$$

Let $y_{0}, \ldots, y_{d}$ denote the dual basis in $V$ to the monomial basis in $W=\mathrm{H}^{0} \mathcal{O}_{\mathbf{P}^{1}}(d)$.

The map $T^{0}(\mathcal{F})=E^{d} \rightarrow E(-1)^{2 d}=T^{1}(\mathcal{F})$ matrix comes from the multiplication $\mathrm{H}^{0}\left(\mathbf{P}^{1}, \mathcal{O}(d-1)\right) \times \mathrm{H}^{0}\left(\mathbf{P}^{1}, \mathcal{O}(d)\right) \rightarrow \mathrm{H}^{0}\left(\mathbf{P}^{1}, \mathcal{O}(2 d-1)\right)$, hence is given by the Sylvester type matrix

$$
B=\left(b_{k l}\right)=\left(y_{k-l}\right)=\operatorname{transpose}\left(\begin{array}{cccccccc}
y_{0} & y_{1} & \ldots & y_{d} & 0 & \ldots & 0 \\
0 & y_{0} & \ldots & y_{d-1} & y_{d} & \ldots & 0 \\
\vdots & \ddots & \ddots & & \ddots & \ddots & \vdots \\
0 & \ldots & 0 & y_{0} & \ldots & y_{d-1} & y_{d}
\end{array}\right)
$$

To prove the formula, we must show that the kernel of this matrix is the image of a matrix $A=\left(a_{i, j}^{\prime}\right)$ with $a_{i, j}^{\prime}=\sum_{\substack{p<\min (i, j) \\ p+q=i+j-1}} y_{p} \wedge y_{q}$.

The equation $B \cdot A=0$ holds since a term $y_{k-l} \wedge y_{p} \wedge y_{q}$ arising in the product $b_{k l} a_{l j}^{\prime}$ is cancelled either by a term $y_{p} \wedge y_{k-l} \wedge y_{q}$ in the product $b_{k, k-p} a_{k-p, j}^{\prime}$ or by a term $y_{q} \wedge y_{p} \wedge y_{k-l}$ in $b_{k, k-q} a_{k-q, j}^{\prime}$, in case $k-l<j$ or $j \leq k-l$, respectively.

Since the $d$ rows of $A$ are linearly independent, and we know that the kernel of $B$ is generated by $d$ independent elements of degree 2 , we see that the rows of $A$ generate the kernel of $B$ as required.

We can obtain the Sylvester formula instead of the Bézout formula by applying $\mathbf{U}_{2}$ to the first shift of the Tate resolution. The resulting complex

$$
\bigoplus^{d} U \rightarrow \bigoplus^{2 d} \mathcal{O}
$$

written in Stiefel coordinates, gives the classical Sylvester formula for two polynomials $f=f_{0} s^{d}+f_{1} s^{d-1} t+\ldots+f_{d} t^{d}$ and $g=g_{0} s^{d}+g_{1} s^{d-1} t+\ldots+g_{d} t^{d}$ of equal degree.

Arbitrary curves. We will generalize these formulas to arbitrary curves over an algebraically closed field, and obtain partial results for more general ground fields.

By a curve we will mean a purely 1-dimensional scheme $X$, projective over $K$. The theory of Ulrich sheaves on curves is significantly simpler than the theory for higher-dimensional varieties because it is essentially independent of the embedding. To state the result, we say that a sheaf $\mathcal{G}$ on a curve $X$ has no cohomology if $\mathrm{H}^{0}(\mathcal{G})=\mathrm{H}^{1}(\mathcal{G})=0$.

Theorem 4.3. If $X$ is a curve embedded in $\mathbf{P}=\mathbf{P}^{n+1}$ with hyperplane divisor $H$, then a sheaf $\mathcal{F}$ is an Ulrich sheaf for $X$ in $\mathbf{P}$ if and only if $\mathcal{F}=\mathcal{G}(H)$ for some $\mathcal{G}$ with no cohomology.

Proof. If $\mathcal{F}$ is Ulrich, then $\mathrm{H}^{0}(\mathcal{F}(-H))=\mathrm{H}^{1}(\mathcal{F}(-H))=0$. Conversely, if $\mathcal{G}=$ $\mathcal{F}(-H)$ has no cohomology, then $\mathcal{F}$ is 0-regular because $\mathrm{H}^{1}(\mathcal{F}(-H))=\mathrm{H}^{1}(\mathcal{G})=0$. Similarly, $\mathcal{E}_{x} t_{\mathbf{P}}^{n-1}\left(\mathcal{F}, \mathcal{O}_{\mathbf{P}}(-n-1)\right)$ is 2-regular because $\mathrm{H}^{1}(\check{\mathcal{F}}(1))=\mathrm{H}^{0}(\mathcal{F}(-1))=$ $\mathrm{H}^{0}(\mathcal{G})=0$. (One can also see the desired vanishing directly from the Tate resolution: For example, the vanishing of $\mathrm{H}^{0}(\mathcal{G})$ implies that the free module $T^{0}(\mathcal{G})$ has no generators in degree 0 ; and it follows that for $j<0$ the module $T^{-j}(\mathcal{G})$ has no 
generators in degree $-j$. But by [Eisenbud et al. 2001, Theorem 7.1] the space of generators of $T^{-j}(\mathcal{G})$ in degree $-j$ is $\mathrm{H}^{0}(\mathcal{G}(-j H))$.

To find sheaves with no cohomology, it suffices to look for sheaves on a single component of the reduced scheme $X_{\text {red }}$ or even on its normalization. Thus we are led to ask: Given a nonsingular irreducible curve $X$ over an arbitrary field $K$, what are the sheaves $\mathcal{G}$ over $X$ with no cohomology? Such a sheaf $\mathcal{G}$ can have no torsion, so (since $X$ is nonsingular) $\mathcal{G}$ is automatically locally free. From the vanishing of the cohomology we see that the Euler characteristic of $\mathcal{G}$ is 0 , so by Riemann-Roch the degree of $\mathcal{G}$ is $\operatorname{rank}(\mathcal{G}) \cdot(g-1)$, where $g=\operatorname{genus}(X)$. Over an algebraically closed field, there are always line bundles of this type. The following proposition generalizes the fact that the equation of any plane curve can be written as the determinant of a linear matrix:

Proposition 4.4. A line bundle $L$ on a nonsingular irreducible curve $X$ has no cohomology if and only if $\operatorname{deg}(L)=\operatorname{genus}(X)-1$ and $L$ has no sections. If $X$ contains infinitely many $K$-rational points, then such line bundles exist on $X$, and thus the Chow form of $X$, in any projective embedding, can be written as a determinant of linear forms in the Plücker coordinates.

Proof. The first statement is immediate from the Riemann-Roch theorem. For the second, take $L=\mathcal{O}_{X}\left(p_{1}+\cdots+p_{g}-q\right)$, where the $p_{i}$ and $q$ are general $K$-rational points.

Corollary 4.5. If $X \subset \mathbf{P}^{n}$ is a 1-dimensional scheme over an arbitrary field, then $X$ has an Ulrich sheaf.

Proof. By Proposition 4.4 the normalization of a component of $X_{\text {red }}$ has an Ulrich line bundle defined over a finite field extension of the ground field, which gives an Ulrich sheaf of higher rank on $X$ defined over the ground field.

To arrive at explicit resultant formulas we have to compute the appropiate differentials in the Tate complex.

Example 4.6 (Hyperelliptic resultant formulas). Consider a fixed polynomial $f=$ $f_{0}+f_{1} t+\cdots+f_{2 g+2} t^{2 g+2}$ with no multiple roots. To write explicit Stiefel and Bézout formulas for the resultant of two functions $a(t)+b(t) \sqrt{f(t)}$ and $c(t)+d(t) \sqrt{f(t)}$ with $a, b, c, d \in K[t]$, we consider them as functions on the hyperellipic curve $C$ of genus $\mathrm{g}$ with function field $K(t, \sqrt{f})$. Let $k=\max \{\operatorname{deg} a, g+1+\operatorname{deg} b, \operatorname{deg} c, g+1+\operatorname{deg} d\}$ and consider the embedding of $C$ given by $t \mapsto\left(1: t: \ldots: t^{k}: \sqrt{f}: t \sqrt{f}: \ldots\right.$ : $\left.t^{k-g-1} \sqrt{f}\right)$. We want the Chow form of this embedding. By Theorem 4.3 and Theorem [3.3, we can express the Chow form as the determinant of a symmetric matrix by using the Ulrich sheaf $\mathcal{L}(H)$, where $\mathcal{L}$ is a line bundle such that $\mathcal{L} \otimes$ $\mathcal{L}=\omega_{C}$, the canonical bundle, and $\mathcal{L}$ has no cohomology. A nonvanishing theta characteristic in turn corresponds to a factorization $f=f^{(1)} f^{(2)}$ of $f$ into two polynomials of degree $g+1$. All of our formulas will depend on the choice of such factorization and we will obtain $\frac{1}{2}\left(\begin{array}{c}2 g+2 \\ g+1\end{array}\right)$ Bézout formulas.

Before we come to the Bézout formulas we will derive a Stiefel formula for the resultant that is highly parallel to the Sylvester formula for the ordinary resultant. We will then deduce a Bézout formula in a way that is analogous to our proof of 
Proposition 4.2, Let

$$
\operatorname{syl}(k, r)=\operatorname{transpose}\left(\begin{array}{ccccccc}
r_{0} & r_{1} & \ldots & r_{k} & 0 & \ldots & 0 \\
0 & r_{0} & \ldots & r_{k-1} & r_{k} & \ldots & 0 \\
\vdots & \ddots & \ddots & & \ddots & \ddots & \vdots \\
0 & \ldots & 0 & r_{0} & \ldots & r_{k-1} & r_{k}
\end{array}\right)
$$

be the $2 k \times k$ "Sylvester block" of a polynomial $r$ of degree $k$.

Proposition 4.7. With notation as above, two functions $a+b \sqrt{f}$ and $c+d \sqrt{f}$ with $a, b, c, d \in K[t]$ have a common zero if and only if the determinant of the $4 k \times 4 k$ matrix

$$
\left(\begin{array}{cccc}
\operatorname{syl}(k, a) & \operatorname{syl}\left(k, b f^{(2)}\right) & \operatorname{syl}(k, c) & \operatorname{syl}\left(k, d f^{(2)}\right) \\
\operatorname{syl}\left(k, b f^{(1)}\right) & \operatorname{syl}(k, a) & \operatorname{syl}\left(k, d f^{(1)}\right) & \operatorname{syl}(k, c)
\end{array}\right)
$$

vanishes.

Proof. Let $\pi: C \rightarrow \mathbf{P}^{1}$ denote the double cover corresponding to the inclusion $K(t) \subset K(C)=K(t)[\sqrt{f}]$. We consider the embedding of $C$ as a curve of degree $2 k$ in projective space $\mathbf{P}^{2 k+1-g}$ corresponding to the line bundle $\mathcal{O}_{C}(H)=\pi^{*}\left(\mathcal{O}_{\mathbf{P}^{1}}(k)\right)$. The space of global sections of $\mathcal{O}_{C}(H)$ has basis corresponding to the functions $1, t, \ldots, t^{k}, \sqrt{f}, t \sqrt{f}, \ldots, t^{k-g-1} \sqrt{f}$, so the Chow form of $C$ in this embedding is the resultant we seek. We write $e_{0}, \ldots, e_{k}, e_{k+1}, \ldots, e_{2 k-g} \in V=\mathrm{H}^{0}\left(\mathcal{O}_{C}(H)\right)^{*}$ for the dual basis.

Every line bundle $\mathcal{L}$ on $C$ can be described as a rank 2 vector bundle $\mathcal{B}=$ $\pi_{*} \mathcal{L}$ on $\mathbf{P}^{1}$ together with an action $\mathcal{B} \stackrel{y}{\rightarrow} \mathcal{B}(g+1)$ satisfying $y^{2}=f \cdot i d_{\mathcal{B}}$. For example $\pi_{*} \mathcal{O}_{C}=\mathcal{O} \oplus \mathcal{O}(-g-1)$ with the action defined by $y=\left(\begin{array}{ll}0 & f \\ 1 & 0\end{array}\right)$. The bundle $\mathcal{B}=\mathcal{O}(-1) \oplus \mathcal{O}(-1)$ with the action of $\left(\begin{array}{cc}0 & f^{(1)} \\ f^{(2)} & 0\end{array}\right)$ corresponds to a nonvanishing theta characteristic $\mathcal{F}$ on $C$. In particular, $\mathcal{F}$ is a line bundle of degree $g-1$ with no cohomology. See Buchweitz and Schreyer 2002 for a detailed exposition. The Stiefel formula above is obtained by applying the functor $\mathbf{U}$ to the line bundle $\mathcal{F}(2 H)$.

The space of global sections of $\mathcal{F}(H)$ has a basis corresponding to the functions

$$
\sqrt{f^{(1)}}, t \sqrt{f^{(1)}}, \ldots, t^{k-1} \sqrt{f^{(1)}}, \sqrt{f^{(2)}}, t \sqrt{f^{(2)}}, \ldots, t^{k-1} \sqrt{f^{(2)}},
$$

while $\mathrm{H}^{0}(\mathcal{F}(2 H))$ has a basis corresponding to

$$
\sqrt{f^{(1)}}, t \sqrt{f^{(1)}}, \ldots, t^{2 k-1} \sqrt{f^{(1)}}, \sqrt{f^{(2)}}, t \sqrt{f^{(2)}}, \ldots, t^{2 k-1} \sqrt{f^{(2)}} .
$$

Thus the map

$$
\operatorname{Hom}\left(E, \mathrm{H}^{0}(\mathcal{F}(H))\right) \rightarrow \operatorname{Hom}\left(E, \mathrm{H}^{0}(\mathcal{F}(2 H))\right)
$$

in the Tate resolution is given by the $4 k \times 2 k$ matrix over the exterior algebra

$$
B=\left(\begin{array}{cc}
\operatorname{syl}\left(k, e_{0}+e_{1} t+\cdots+e_{k} t^{k}\right) & \operatorname{syl}\left(k,\left(e_{k+1}+\cdots+e_{2 k-g} t^{k-g-1}\right) f^{(2)}\right) \\
\operatorname{syl}\left(k,\left(e_{k+1}+\cdots+e_{2 k-g} t^{k-g-1}\right) f^{(1)}\right) & \operatorname{syl}\left(k, e_{0}+e_{1} t+\cdots+e_{k} t^{k}\right)
\end{array}\right) .
$$

The desired Sylvester formula follows by interpreting the induced map

$$
\mathrm{H}^{0}(\mathcal{F}(H)) \otimes U \rightarrow \mathrm{H}^{0}(\mathcal{F}(2 H)) \otimes \mathcal{O}_{\mathbf{G}}
$$

in terms of Stiefel coordinates. 
We now use these constructions as in Proposition 4.2 to derive hyperelliptic Bézout formulas. It suffices to compute the kernel of the map $B$ of Proposition 4.7 By Theorem 0.1 this will be a $2 k \times 2 k$ matrix with entries in $\Lambda^{2} V$. Because $\mathcal{F}$ is a theta characteristic, Theorem 3.3 shows that the kernel will be represented in suitable bases by a symmetric matrix.

The final formula may be written in terms of the $2 \times 2$ minors of the $2 \times(2 k+1-g)$ matrix

$$
\left(\begin{array}{llllll}
a_{0} & \ldots & a_{k} & b_{0} & \ldots & b_{k-g-1} \\
c_{0} & \ldots & c_{k} & d_{0} & \ldots & d_{k-g-1}
\end{array}\right) .
$$

However we will work with the larger $2 \times 3(k+1)$ matrix

$$
\left(\begin{array}{ccccccccc}
a_{0} & \ldots & a_{k} & \left(b f^{(1)}\right)_{0} & \ldots & \left(b f^{(1)}\right)_{k} & \left(b f^{(2)}\right)_{0} & \ldots & \left(b f^{(2)}\right)_{k} \\
c_{0} & \ldots & c_{k} & \left(d f^{(1)}\right)_{0} & \ldots & \left(d f^{(1)}\right)_{k} & \left(d f^{(2)}\right)_{0} & \ldots & \left(d f^{(2)}\right)_{k}
\end{array}\right)
$$

whose minors are linear combinations of those of the matrix above, with coefficients that depend on the coefficients of $f^{(1)}$ and $f^{(2)}$.

If $0 \leq p, q \leq k$, then we denote by $[p, q]$ the minor formed by the columns with indices $p$ and $q$. We write $p^{(1)}$ for the column with index $p+(k+1)$ and $q^{(2)}$ for the column with index $q+2(k+1)$. Thus brackets like $\left[p^{(1)}, q\right]$ and $\left[p^{(1)}, q^{(2)}\right]$ represent $2 \times 2$ minors of the large matrix.

Consider the $k \times k$ matrices $A^{11}, \ldots, A^{22}$ defined by

$$
\begin{aligned}
& A_{i, j}^{11}=\sum_{\substack{0 \leq p<q \leq k \\
p<\min (i, j) \\
p+q=i+j-1}}\left[p^{(2)}, q\right]+\left[p, q^{(2)}\right], \\
& A_{i, j}^{12}=\sum_{\substack{0 \leq p<q \leq k \\
p<\min (i, j) \\
p+q=i+j-1}}[p, q]+\sum_{\substack{0 \leq p, q \leq k \\
p \leq \leq \\
p+q=i+j-1}}\left[p^{(1)}, q^{(2)}\right], \\
& A_{i, j}^{21}=\sum_{\substack{0 \leq p<q \leq k \\
p<\ln (i, j) \\
p+q=i+j-1}}[p, q]+\sum_{\substack{0 \leq p, q \leq k \\
p, s \leq k \\
p+q=i+j-1}}\left[p^{(2)}, q^{(1)}\right], \\
& A_{i, j}^{22}=\sum_{\substack{0 \leq p<q \leq k \\
p<\min (i, j) \\
p+q=i+j-1}}\left[p^{(1)}, q\right]+\left[p, q^{(1)}\right] .
\end{aligned}
$$

The matrix

$$
A=\left(\begin{array}{ll}
A^{11} & A^{12} \\
A^{21} & A^{22}
\end{array}\right)
$$

is actually symmetric. This becomes visible if we expand the expressions into brackets of the smaller $2 \times(2 k+1-g)$ matrix.

Proposition 4.8. Suppose $k \leq 12$. The functions $a+b \sqrt{f}$ and $c+d \sqrt{f}$ have $a$ common zero if and only if the determinant of the matrix $A$ vanishes.

Proof. As in the proof of Proposition 4.2 it suffices to check that $B \cdot A=0$, when we regard $A$ as a matrix over the exterior algebra, because the linear independence of the columns of $A$ is visible from the specialization to the case of binary forms $b=d=0$. For each specific value of $g$ and $k$ this can be checked by computer, and we did this for all cases $1 \leq g+1 \leq k \leq 12$.

The formula should certainly hold for any $k$; but as noted in the proof, we have performed the necessary computations only up to $k=12$. 
Notice that in case $b=d=0$ the matrix reduces to twice the Bezout matrix for binary forms of degree $k$. This fits with the fact that two functions on $\mathbf{P}^{1}$ with a common zero have two common zeroes when pulled back to $C$.

As a concrete application of Proposition 4.8 we do the case of an elliptic curve over the complex numbers.

Example 4.9 (Resultant of doubly periodic functions). Consider an elliptic curve $C=\mathbf{C} / \Gamma$ and the corresponding Weierstrass $\wp$-function, with functional equation

$$
\wp^{\prime}(z)=4 \wp^{3}(z)-g_{2} \wp(z)-g_{3}=4\left(\wp(z)-\rho_{1}\right)\left(\wp(z)-\rho_{2}\right)\left(\wp(z)-\rho_{3}\right)
$$

where the $\rho_{j}$ are the values of $\wp$ at the half periods.

Corollary 4.10. Two doubly periodic functions

$$
f(z)=a_{0}+a_{1 \wp}(z)+a_{2} \wp^{2}(z)+b_{0} \wp^{\prime}(z) / 2
$$

and

$$
g(z)=c_{0}+c_{1} \wp(z)+c_{2} \wp^{2}(z)+d_{0} \wp^{\prime}(z) / 2
$$

have a common zero if and only if the determinant of

$$
\left(\begin{array}{cccc}
-\rho_{1} \rho_{2}[13]-\left(\rho_{1}+\rho_{2}\right)[03] & -\rho_{1} \rho_{2}[23]+[03] & {[01]} & {[02]} \\
-\rho_{1} \rho_{2}[23]+[03] & \left(\rho_{1}+\rho_{2}\right)[23]+[13] & {[02]} & {[12]} \\
{[01]} & {[02]} & \rho_{3}[13]+[03] & \rho_{3}[23] \\
{[02]} & {[12]} & \rho_{3}[23] & -[23]
\end{array}\right)
$$

vanishes, where the bracket $[i j]$ denotes the minor made from the $i$-th and $j$-th columns of the matrix

$$
\left(\begin{array}{llll}
a_{0} & a_{1} & a_{2} & b_{0} \\
c_{0} & c_{1} & c_{2} & d_{0}
\end{array}\right) .
$$

Proof. This formula follows from Proposition 4.8 with one of the roots of $f$ at infinity and with the factorization given by $f^{(1)}=\left(\wp(z)-\rho_{1}\right)\left(\wp(z)-\rho_{2}\right)$.

Returning to our general discussion, we may ask whether it is possible to give a Bézout formula for the Chow form of a curve over a field $K$ even if the curve does not have enough $K$-rational points to apply Theorem 4.3. In this case the curve may have no rank 1 Ulrich sheaf, as happens, for example, for a conic without real points in $\mathbf{P}_{\mathbf{R}}^{2}$. However, it may be that there are always rank 2 Ulrich sheaves. For example, assuming that $X$ has genus 0 , the structure sheaf $\mathcal{O}_{X}$ and the canonical bundle $\omega_{X}$ are defined over $K$, and there is the unique extension

$$
\eta: \quad 0 \rightarrow \omega_{X} \rightarrow \mathcal{E} \rightarrow \mathcal{O}_{X} \rightarrow 0
$$

corresponding to a nonzero element $\eta \in \mathrm{H}^{1}\left(\omega_{X}^{-1}\right)=K$. Over an algebraic closure of $K$ the bundle $\mathcal{E}$ splits as $\mathcal{O}_{\mathbf{P}^{1}}(-1) \oplus \mathcal{O}_{\mathbf{P}^{1}}(-1)$ (the sequence above is the Koszul complex) and thus $\mathcal{E}$ has no cohomology.

The main theorem of [Brennan et al. 1987] generalizes this example and says that if $K$ is algebraically closed and $X$ is a 1-dimensional arithmetically CohenMacaulay subscheme of $\mathbf{P}$, then there exists a rank 2 sheaf $\mathcal{F}$ with no cohomology, which in addition satisfies $\mathcal{F} \cong \mathcal{H} o m\left(\mathcal{F}, \omega_{X}\right)$. (Their statement does not include the separability hypothesis below; but they apply a result of [Eisenbud 1988] which is proved only in the algebraically closed case. We do not see how to extend their proof beyond the separable case.) A variation on their proof allows one to drop the "arithmetically Cohen-Macaulay" hypothesis. Here is a geometric version of the argument, developed in conversation with Joe Harris. 
Proposition 4.11. Let $X$ be a projective curve, separable over the field $K$. If $K$ is infinite, then $X$ has a coherent sheaf $\mathcal{E}$ with no cohomology that is a rank 2 vector bundle over the normalization of $X_{\mathrm{red}}$ and satisfies $\mathcal{H o m}\left(\mathcal{E}, \omega_{X}\right)=\mathcal{E}$ skewsymmetrically.

Proof. Let $\pi: C \rightarrow X_{\text {red }}$ be the normalization. It is enough to find a rank 2 vector bundle without cohomology on $C$ with $\mathcal{H o m}\left(\mathcal{E}, \omega_{C}\right)=\mathcal{E}$, because we have $\mathcal{H o m}_{X}\left(\mathcal{E}, \omega_{X}\right)=\mathcal{H o m}_{C}\left(\mathcal{E}, \mathcal{H o m}\left(\mathcal{O}_{C}, \omega_{X}\right)\right)=\mathcal{H o m}_{C}\left(\mathcal{E}, \omega_{C}\right)$. Since we have dealt with the case of $\mathbf{P}^{1}$ above, we will assume that the genus $g$ of $C$ is greater than 0 . Let $L$ be a line bundle on $C$ of strictly positive degree.

Any extension class $\eta \in \operatorname{Ext}^{1}\left(\omega_{C} \otimes L, L^{-1}\right)$ gives rise to a short exact sequence

$$
\eta: \quad 0 \rightarrow L^{-1} \rightarrow \mathcal{E} \rightarrow \omega_{C} \otimes L \rightarrow 0
$$

where $\mathcal{E}$ is a vector bundle. For any such bundle $\bigwedge^{2} \mathcal{E}=\omega_{C}$, whence $\mathcal{H o m}\left(\mathcal{E}, \omega_{C}\right)=$ $\mathcal{E}$ skew-symmetrically.

By Serre duality $\chi(\mathcal{E})=0$, so $\mathcal{E}$ will be an Ulrich sheaf as long as $\mathrm{H}^{0}(\mathcal{E})=$ 0 . Since $\mathrm{H}^{0}\left(\mathcal{L}^{-1}\right)=0$, this condition is satisfied if and only if the connecting homomorphism

$$
\delta_{\eta}: \mathrm{H}^{0}\left(\omega_{C} \otimes L\right) \rightarrow \mathrm{H}^{1}\left(\mathcal{L}^{-1}\right)=\mathrm{H}^{0}\left(\omega_{C} \otimes L\right)^{*}
$$

is an isomorphism. But

$$
\eta \in \operatorname{Ext}^{1}\left(\omega_{C} \otimes L, L^{-1}\right) \cong \mathrm{H}^{1}\left(L^{-2} \otimes \omega_{C}^{-1}\right) \cong \mathrm{H}^{0}\left(L^{2} \otimes \omega_{C}^{2}\right)^{*},
$$

and $\delta_{\eta}$ is induced by the multiplication pairing

$$
\mathrm{H}^{0}\left(L \otimes \omega_{C}\right) \otimes \mathrm{H}^{0}\left(L \otimes \omega_{C}\right) \stackrel{m}{\longrightarrow} \mathrm{H}^{0}\left(L^{2} \otimes \omega_{C}^{2}\right)
$$

in the sense that $\eta$ goes to $\delta_{\eta}$ under the composition

$$
\begin{aligned}
\mathrm{H}^{0}\left(L^{2} \otimes \omega_{C}^{2}\right)^{*} \stackrel{m^{*}}{\longrightarrow} & \mathrm{H}^{0}\left(L \otimes \omega_{C}\right)^{*} \otimes \mathrm{H}^{0}\left(L \otimes \omega_{C}\right)^{*} \\
& \cong \mathrm{H}^{0}\left(L \otimes \omega_{C}\right)^{*} \otimes \mathrm{H}^{1}\left(L^{-1}\right) \\
& \cong \operatorname{Hom}\left(\mathrm{H}^{0}\left(L \otimes \omega_{C}\right), \mathrm{H}^{1}\left(L^{-1}\right)\right) .
\end{aligned}
$$

The ring $R=\bigoplus_{d} \mathrm{H}^{0}\left(L^{d} \otimes \omega_{C}^{d}\right)$ is an integral domain. By separability, it splits into a product of integral domains over the algebraic closure of $K$. It follows that the multiplication pairing is a direct sum of 1-generic pairings in the sense of Eisenbud 1988. The results of that paper show that $\delta_{\eta}$ is an isomorphism unless $\eta$ lies in a certain proper hypersurface in $\mathrm{H}^{0}\left(L^{2} \otimes \omega_{C}^{2}\right)$. If $K$ is infinite, then this hypersurface cannot contain all the $K$-rational points of this vector space.

Example 4.12 (Pointless conics). The conic $C \subset \mathbf{P}^{2}$ defined by $x^{2}+y^{2}+z^{2}=0$ has no line bundle of degree -1 defined over $\mathbf{R}$. However there are rank 2 Ulrich sheaves. The cokernel

$$
\mathcal{F}=\operatorname{coker}\left(\mathcal{O}_{\mathbf{P}^{2}}^{4}(-2) \stackrel{M}{\longrightarrow} \mathcal{O}_{\mathbf{P}^{2}}^{4}(-1)\right)
$$

given by the matrix

$$
M=\left(\begin{array}{cccc}
0 & x & y & z \\
-x & 0 & z & -y \\
-y & -z & 0 & x \\
-z & y & -x & 0
\end{array}\right)
$$

is a rank 2 sheaf on $C$ with no cohomology. An explicit Bézout formula for the Chow form of $C$ can be derived from the Pfaffian Bézout formula for the resultant of three 
quadratic forms in three variables given in the introduction, by specializing one of the three quadratic forms to $x^{2}+y^{2}+z^{2}$ and eliminating unnecessary variables.

\section{Resultant formulas and Veronese embeddings}

Generalizing the case of binary forms in another direction, we consider the resultant of $k+1$ forms of degree $d$ in $k+1$ variables. That is, we consider the Chow form of the $d$-uple embedding

$$
\mathbf{P}^{k} \hookrightarrow \mathbf{P}^{N}
$$

with $N=\left(\begin{array}{c}d+k \\ k\end{array}\right)-1$.

To find determinantal or Pfaffian formulas for powers of such Chow forms, we need to look for vector bundles on $\mathbf{P}^{k}$ that become Ulrich sheaves on the $d$-uple embedding. Similarly, Stiefel formulas come from weakly Ulrich sheaves. By an argument shown to us by Jerzy Weyman, Ulrich sheaves always exist, but we shall see that in some cases all Ulrich sheaves have very high rank.

By way of comparison, the classical search for Bézout or Stiefel formulas was essentially a search for line bundles on $\mathbf{P}^{k}$ that become Ulrich or weakly Ulrich on the $d$-uple embedding. Weakly Ulrich line bundles exist (and were found classically, e.g., Gel'fand et al. 1994, Chap 13, Prop. 1.6] if and only if $k \leq 3$ or $k=4, d \leq 3$ or $k=5, d=2$ (Ulrich line bundles never exist except when $k \leq 2$ or $d=1$ ). We get a few more Stiefel formulas for the resultants themselves (and not just powers) from the Horrocks-Mumford bundle in the case $k=5, d=4,6$ or 8 .

It turns out that the cohomology of a sheaf that becomes an Ulrich sheaf on the $d$-uple embedding of $\mathbf{P}^{k}$ is determined by the rank of the sheaf alone, and the same idea works for the $d$-uple embedding of any variety:

Theorem 5.1. Let $\iota: \mathbf{P}^{m} \hookrightarrow \mathbf{P}^{n}$ be the d-uple embedding. Suppose $\mathcal{F}$ is a sheaf of dimension $k$ on $\mathbf{P}^{m}$. The sheaf $\iota_{*} \mathcal{F}$ is an Ulrich sheaf on $\mathbf{P}^{n}$ if and only if

$$
h^{i}(\mathcal{F}(e)) \neq 0 \Leftrightarrow \begin{cases}i=0, & -d<e \\ 0<i<k, & -(i+1) d<e<-i d \\ i=k, & e<-k d .\end{cases}
$$

In particular, $\mathcal{F}$ then has natural cohomology as a sheaf on $\mathbf{P}^{m}$. Thus all the $h^{i}(\mathcal{F}(e))$ are determined by the formula

$$
\chi(\mathcal{F}(e))=h^{0}(\mathcal{F})\left(\begin{array}{c}
\frac{e}{d}+k \\
k
\end{array}\right) .
$$

If $\mathcal{F}$ is a vector bundle of rank $r$ on $\mathbf{P}^{m}$, then we can rewrite this formula as $\chi(\mathcal{F}(e))=\frac{r}{m !}(e+d) \cdots(e+m d)=\left(\frac{r}{m !} e^{m}\right)+\cdots+r d^{m}$.

The vanishing and nonvanishing results in the first part of Theorem 5.1 have a very simple interpretation in terms of the Betti diagram of the Tate resolution of $\mathcal{F}$ : They say that the nonzero terms form a sequence of nonoverlapping strands and that all of the strands representing intermediate cohomology have length precisely $d-1$. The formulas in the second part then give the values of the nonzero terms. For example, if $\mathcal{F}$ is a rank 2 vector bundle on $\mathbf{P}^{2}$ which is an Ulrich sheaf for the $d$-uple embedding, Theorem 5.1 says precisely that the Tate resolution of $\mathcal{F}$, considered as a sheaf on $\mathbf{P}^{2}$, has Betti diagram

$$
\begin{aligned}
& \begin{array}{lllllllllllll}
\cdots & 2(d+2) & 1(d+1) & 0 & 0 & 0 & 0 & 0 & 0 & 0 & \cdots & 0 & \cdots
\end{array} \\
& \begin{array}{lllllllllllll}
\ldots & 0 & 0 & 1(d-1) & 2(d-2) & \cdots & (d-2) 2 & (d-1) 1 & 0 & 0 & \cdots & 0 & \ldots
\end{array} \\
& \begin{array}{lllllllllllllll}
\cdots & 0 & 0 & 0 & 0 & 0 & 0 & 0 & 1(d+1) & 2(d+2) & \cdots & d(2 d) & \cdots
\end{array}
\end{aligned}
$$


where the zero-th term is the one occuring at the far right (so that, for example, $h^{0}(\mathcal{F})=2 d^{2}$ ). Further examples are given in the discussion of sheaves on $\mathbf{P}^{3}$ below.

To prove that the cohomology vanishes as we claim, we will repeatedly use the following elementary result, which is an easy case of Eisenbud et al. 2001, Lemma 7.4]. For the reader's convenience we give a quick proof.

Lemma 5.2. Suppose $\mathcal{G}$ is a sheaf on $\mathbf{P}^{k}$.

(a) If $\mathrm{H}^{i+j}(\mathcal{G}(-1-j))=0$ for all $j \geq 0$, then $\mathrm{H}^{i}(\mathcal{G})=0$.

(b) If $\mathrm{H}^{i-j}(\mathcal{G}(1+j))=0$ for all $j \geq 0$, then $\mathrm{H}^{i}(\mathcal{G})=0$.

Note that the case $i=1$ in part (a) is Mumford's result showing that a (-1)regular sheaf is 0 -regular.

Proof of Lemma5.2 (a): Translating the condition in (a) to a condition on the Tate resolution $T^{\bullet}(\mathcal{G})$ over the exterior algebra $E$, we see that the free summand $\mathrm{H}^{i}(\mathcal{G}) \otimes \omega_{E}$ in $T^{0}(\mathcal{G})$ maps injectively into $T^{1}(\mathcal{G})$. Since $T^{\bullet}(\mathcal{G})$ is a minimal complex and $E$ is Artinian, this is impossible unless $\mathrm{H}^{i}(\mathcal{G})=0$.

Part (b) follows by applying the same argument to the dual of the Tate resolution.

Proof of Theorem 5.1. We first show, by induction on $i$, that for $i<k$, we have $\mathrm{H}^{i}(\mathcal{F}(e))=0$ if $e \leq-(i+1) d$. Since $\mathcal{F}$ becomes an Ulrich sheaf under the $d$-uple embedding, we have $\mathrm{H}^{0}(\mathcal{F}(-d))=0$, and it follows that $\mathrm{H}^{i}(\mathcal{F}(e))=0$ for $e \leq-d$, which is the case $i=0$. For $i>0$ we proceed by descending induction on $e$. Again since $\mathcal{F}$ becomes Ulrich on the $d$-uple embedding, we have $\mathrm{H}^{i}(\mathcal{F}(-(i+1) d))=0$, the initial case. Assuming that $\mathrm{H}^{i}(\mathcal{F}(e))=0$ for some $e<-(i+1) d$, the induction on $i$ gives the hypothesis to apply part (b) of Lemma[5.2, showing that $\mathrm{H}^{i}(\mathcal{F}(-e-1))$ vanishes.

Similarly, $\mathrm{H}^{i}(\mathcal{F}(e))=0$ for $i>0$ and $e \geq-i \cdot d$ follows by induction and part (b) of Lemma 5.2. The nonvanishing of the remaining cohomology follows, since otherwise the Tate resolution for $\mathcal{F}$ would contain terms equal to zero.

We next prove the formulas for $\chi(\mathcal{F}(e))$. If $\iota_{*} \mathcal{F}$ is an Ulrich sheaf, Corollary 2.2 shows that $\chi(\mathcal{F}(d t))=h^{0}(\mathcal{F})\left(\begin{array}{c}k+t \\ k\end{array}\right)$. Since $\chi(\mathcal{F}(t))$ is a polynomial, it is determined by this relation, yielding the first formula.

If in addition $\mathcal{F}$ is a bundle of rank $r$ on $\mathbf{P}^{m}$, so that $k=m$, then part (c) of Proposition 2.1 shows that $\mathrm{h}^{0}(\mathcal{F})=\operatorname{deg} \iota_{*}(\mathcal{F})$, which is $r$ times the degree of the $d$-uple embedding of $\mathbf{P}^{m}$, that is, $\mathrm{h}^{0}(\mathcal{F})=r d^{m}$. Substituting this in the first formula, we get the last formulas. (One could also argue directly from the fact that the last formula must be a polynomial of degree $m$ which vanishes at $-n d$ for $n=1, \ldots, m)$.

Corollary 5.3. Suppose there exists a rank $r$ sheaf on $\mathbf{P}^{k}$ which is an Ulrich sheaf for the d-uple embedding. If a prime $p$ divides $d$ and $p^{t}$ divides $k$ !, then $p^{t}$ divides $r$. For example, any Ulrich sheaf on the $k$ !-uple embedding of $\mathbf{P}^{k}$ has rank a multiple of $k$ !.

Proof. In Theorem 5.1, note that $\chi(\mathcal{F}(1))$ is an integer.

The next result shows that if $X \subset \mathbf{P}^{n}$ is the support of an Ulrich (or weakly Ulrich) sheaf, then the general problem of finding (weakly) Ulrich sheaves for the Veronese embeddings of $X$ can be reduced to the problem for projective spaces. 
Proposition 5.4. Let $X \subset \mathbf{P}^{n}$ be a purely $k$-dimensional scheme, and let $\mathcal{F}$ be an Ulrich sheaf whose support is $X$. Suppose that $\pi: X \rightarrow \mathbf{P}^{k}$ is a finite linear projection. If $\mathcal{E}$ is a sheaf on projective space that is (weakly) Ulrich for the $d$ uple embedding of projective space, then $\mathcal{F} \otimes \pi^{*} \mathcal{E}$ is (weakly) Ulrich for the d-uple embedding of $X$.

Note that a finite linear projection always exists if the base field is infinite - this is "Noether normalization".

Proof. Since the cohomology of $\pi_{*} \mathcal{F}(n)$ is the same as the cohomology of $\mathcal{F}(n)$, we see from the cohomological characterization of Ulrich sheaves that $\pi_{*} \mathcal{F}$ is a trivial bundle $\mathcal{O}_{\mathbf{P}^{k}}^{t}$ on $\mathbf{P}^{k}$. Since

$$
\mathrm{H}^{q}\left(\mathcal{F} \otimes \pi^{*} \mathcal{E}(d)\right)=\mathrm{H}^{q}\left(\pi_{*} \mathcal{F} \otimes \mathcal{E}(d)\right)
$$

this group vanishes for exactly the same values of $q, d$ as does $\mathrm{H}^{q}(\mathcal{E}(d))$, and this determines the weakly Ulrich and Ulrich properties.

If we apply Proposition 5.4 in the case where $X \cong \mathbf{P}^{k}$, embedded by the $e$-uple embedding, we get a weak converse to Corollary 5.3 .

Corollary 5.5. If $\mathbf{P}^{k}$ has Ulrich sheaves of ranks $a$ and $b$ on its $d$-uple and $e$ uple embeddings, respectively, then it has an Ulrich sheaf of rank ab on its de-uple embedding.

Proposition 5.4 and Corollary 5.5 were inspired by the proof of the existence of rank 4 Ulrich sheaves on the 4-uple embedding of $\mathbf{P}^{3}$ given by Douglas Hanes in his thesis [Hanes 2000].

If our ground field $K$ has characteric zero, then up to twists any indecomposable homogenous bundle on $\mathbf{P}^{n}$ can be obtained by applying a Schur functor $S_{\lambda}$ to the universal rank $n$ quotient bundle $Q=\operatorname{coker}\left(\mathcal{O}_{\mathbf{P}^{n}}(-1) \rightarrow \mathcal{O}_{\mathbf{P}^{n}}^{n+1}\right)$ of $\mathbf{P}^{n}$ (the tangent bundle tensor $\left.\mathcal{O}_{\mathbf{P}^{n}}(-1)\right)$. Here $\lambda=\left(\lambda_{1}, \ldots, \lambda_{n}\right)$ is a partition into at most $n$ parts. Note $\left(S_{\lambda} Q\right)(1)=S_{\lambda+(1,1, \ldots, 1)} Q$ and $\mathrm{H}^{0}\left(S_{\lambda} Q\right)=S_{\lambda} V$ with $V=H^{0}(\mathcal{O}(1))^{*}$. Thus up to twist we may assume that $\lambda_{n}=0$. The theorem below implies that $S_{\lambda} Q$ has Castelnuovo-Mumford regularity precisely zero if and only if $\lambda_{n}=0$. For our purposes it is convenient to visualize the partition as a Ferrers diagram whose row lengths are given by the $\lambda_{i}$, as follows:

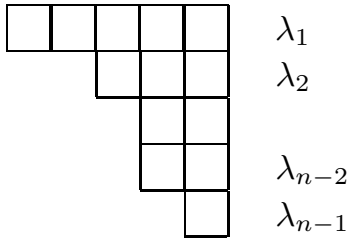

The following result was pointed out to us by J. Weyman.

Theorem 5.6. Suppose that $K$ has characteristic zero. Let $\lambda=\left(\lambda_{1}, \ldots, \lambda_{n-1}\right)$ be a partition and $Q$ the universal rank $n$ quotient bundle on $\mathbf{P}^{n}$. The Tate resolution of the homogeneous bundle $\mathcal{F}=S_{\lambda} Q$ has nonzero terms only in the degrees marked * in the following Betti diagram, in which the Ferrers diagram has shape $\lambda$ as above: 


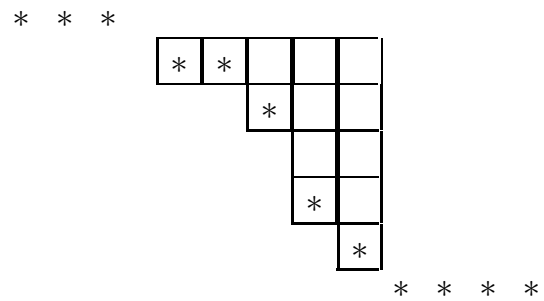

More precisely, for $1 \leq i \leq n-1$ the cohomology group $\mathrm{H}^{i}\left(\left(S_{\lambda} Q\right)(m)\right)$ is nonzero if and only if $\lambda_{n-i+1}<-m-i \leq \lambda_{n-i}, \mathrm{H}^{0} S_{\lambda} Q(m)=0$ if and only if $m<0$ and $\mathrm{H}^{n} S_{\lambda} Q(m)=0$ if and only if $m \geq-n-\lambda_{1}-1$.

Proof. The cohomology of a homogeneous bundle on the homogeneous space

$$
\mathbf{P}^{n}=\mathrm{GL}(n+1) /\left(\begin{array}{cc}
\mathrm{GL}(n) & * \\
0 & \mathrm{GL}(1)
\end{array}\right)
$$

is determined by Bott's formula; see [Jantzen 1987]. In particular $\mathrm{H}^{i} S_{\lambda} Q(m) \neq$ 0 for at most one $i$, and

$$
\mathrm{H}^{i} S_{\lambda} Q(m)=0 \text { for all } i \Leftrightarrow-m \in\left\{\lambda_{i}+n+1-i \mid i=1, \ldots, n\right\} .
$$

Thus the Hilbert polynomial $\chi S_{\lambda} Q(m)$ has precisely $n$ integral zeroes and the Tate resolution "steps down" precisely at these $n$ values by Theorem 5.1

Corollary 5.7. Suppose that $K$ has characteristic zero. The unique indecomposable homogeneous bundle on $\mathbf{P}^{n}$ that is an Ulrich sheaf for the d-uple embedding is $S_{\lambda} Q$

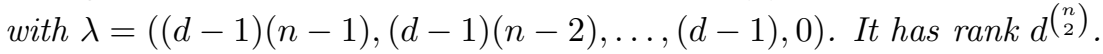

Proof. The first statement follows easily from the previous theorem. The rank of $S_{\lambda} Q$ is given by the hook formula (see [Stanley 1971] or [Fulton 1997, p. 55])

$$
\operatorname{rank} S_{\lambda} Q=\prod_{(i, j) \in \lambda} \frac{n+i-j}{h(i, j)},
$$

where $h(i, j)$ denotes the hook length of the $(i, j)$-th box. The largest hook length is $h(1,1)=(d-1)(n-1)+(n-2)=d(n-1)-1$. The denominators of the first row contribute

$$
\begin{aligned}
\prod_{j} h(1, j)= & (d(n-1)-1)(d(n-1)-2) \\
& \cdot \ldots \cdot(d(n-1)-d+1)(d(n-2)-1) \cdot \ldots \cdot 1 \\
= & \frac{[d(n-1)] !}{d^{n-1}(n-1) !} .
\end{aligned}
$$

The numerators give $\frac{[d(n-1)] !}{(n-1) !}$. Thus the first row contributes $d^{n-1}$ and the rank is $d^{\left(\begin{array}{c}n-1 \\ 2\end{array}\right)+n-1}=d^{\left(\begin{array}{c}n \\ 2\end{array}\right)}$ by induction on the number of rows in $\lambda$.

Chow forms from line bundles on projective spaces. All the classically known formulas (and no new ones) for the resultant of $k+1$ forms of degree $d$ in $k+1$ variables come from applying these ideas to line bundles on projective spaces. These are rarely Ulrich bundles, and we get Bézout formulas in this way only for binary forms of any degree or linear forms in any number of variables. 
On the other hand $\mathcal{L}=\mathcal{O}(j)$ on $\mathbf{P}^{k}$ gives rise to a two term complex, and hence a Stiefel formula for the Chow form of the $d$-uple image, if and only if

$$
\mathrm{H}^{0} \mathcal{L}(-H)=0 \quad \text { and } \quad \mathrm{H}^{k} \mathcal{L}(-(k-2) H)=0,
$$

equivalently, if and only if

$$
d-1 \geq j \geq-k+(k-2) d=(k-2)(d-1)-2 .
$$

Thus the Chow forms of $\mathbf{P}^{1}, \mathbf{P}^{2}, \mathbf{P}^{3}$ for arbitrary $d$-uple embeddings, on $\mathbf{P}^{4}$ for quadrics and cubics and on $\mathbf{P}^{5}$ for quadrics, can be written as determinants of maps of vector bundles on the Grassmannian, or as determinantal formulas in the Stiefel coordinates. This is precisely the list of Gel'fand et al. 1994, Chap. 13, Prop. 1.6]. For instance, in the case of three ternary quadrics we have:

Example 5.8. For the 2-uple embedding (quadrics) of $\mathbf{P}^{2}$ the line bundle $\mathcal{O}_{\mathbf{P}^{2}}(1)$ is weakly Ulrich, and we see that the Chow form is the determinant of a canonical map on the Grassmannian $\mathbf{G}$

$$
\mathcal{O}_{\mathbf{G}}(-1)^{6} \rightarrow U \oplus \mathcal{O}_{\mathbf{G}}^{3}
$$

with $U$ the universal subbundle on $\mathbf{G}=\mathbf{G}_{3}=\mathbf{G}(3, W)$ and $W=\mathrm{H}^{0}\left(\mathcal{O}_{\mathbf{P}^{2}}(2)\right)$. The map is easy to calculate, for example, using the computer algebra system Macaulay2, as a second syzygy matrix over $E$ of the "multiplication" map $\mathrm{H}^{0}\left(\mathcal{O}_{\mathbf{P}^{2}}(3)\right) \rightarrow \mathrm{H}^{0}\left(\mathcal{O}_{\mathbf{P}^{2}}(5)\right) \otimes W^{*}$. In Stiefel coordinates we obtain the matrix

$$
\text { transpose }\left(\begin{array}{cccccc}
a_{0} & b_{0} & c_{0} & {[0,1,5]} & 0 & {[0,1,2]} \\
a_{1} & b_{1} & c_{1} & {[0,3,5]} & {[0,3,4]} & {[0,1,4]-[0,2,3]} \\
a_{2} & b_{2} & c_{2} & {[0,4,5]-[1,2,5]} & {[0,3,5]} & {[0,1,5]} \\
a_{3} & b_{3} & c_{3} & 0 & {[1,3,4]} & {[0,3,4]} \\
a_{4} & b_{4} & c_{4} & {[2,3,5]} & {[2,3,4]+[1,3,5]} & {[0,3,5]} \\
a_{5} & b_{5} & c_{5} & {[2,4,5]} & {[2,3,5]} & 0
\end{array}\right) .
$$

Thus the determinant of this matrix is the resultant of three quadratic forms $d=$ $d_{0} x^{2}+d_{1} x y+d_{2} x z+d_{3} y^{2}+d_{4} y z+d_{5} z^{2}$ for $d=a, b, c$ with $(i, j, k)$-th Plücker coordinate

$$
[i, j, k]=\operatorname{det}\left(\begin{array}{ccc}
a_{i} & b_{i} & c_{i} \\
a_{j} & b_{j} & c_{j} \\
a_{k} & b_{k} & c_{k}
\end{array}\right) .
$$

Ulrich sheaves on $\mathbf{P}^{2}$. To get new formulas for resultants, we replace line bundles with vector bundles of higher rank. The Chow forms of these bundles are the desired resultants raised to a power equal to the rank of the bundle. But if the rank of the bundle is 2 , then its natural symplectic structure allows us to find a polynomial square root by taking a Pfaffian in place of a determinant, so we get formulas for the resultant itself.

Proposition 5.9. If $\alpha$ is a $(d+1) \times(d-1)$ matrix of linear forms on $\mathbf{P}^{2}$ whose minors of order $d-1$ generate an ideal of codimension 3 (the generic value), then

$$
\operatorname{coker}\left(\mathcal{O}_{\mathbf{P}^{2}}(d-2)^{d-1} \stackrel{\alpha}{\rightarrow} \mathcal{O}_{\mathbf{P}^{2}}(d-1)^{d+1}\right)
$$

is an Ulrich sheaf on the d-uple embedding of $\mathbf{P}^{2}$. 
For example, we may take

$$
\alpha=\left(\begin{array}{cccccc}
x_{0} & x_{1} & x_{2} & 0 & \ldots & 0 \\
0 & x_{0} & x_{1} & x_{2} & & \vdots \\
\vdots & & \ddots & \ddots & \ddots & \\
0 & \ldots & & x_{0} & x_{1} & x_{2}
\end{array}\right) .
$$

Proof of Proposition 5.9. Setting $\mathcal{F}=\operatorname{coker}\left(\mathcal{O}_{\mathbf{P}^{2}}(d-2)^{d-1} \stackrel{\alpha}{\rightarrow} \mathcal{O}_{\mathbf{P}^{2}}(d-1)^{d+1}\right)$, we see that $\bigwedge^{2} \mathcal{F} \cong \mathcal{O}_{\mathbf{P}^{2}}(3 d-3)=\mathcal{O}_{\mathbf{P}^{2}}(3 d) \otimes \omega_{\mathbf{P}^{2}}$. Since $\mathcal{F}$ is a rank 2 vector bundle,

$$
\mathcal{F}=\mathcal{F}^{*} \otimes \bigwedge^{2} \mathcal{F}=\mathcal{F}^{*} \otimes \mathcal{O}_{\mathbf{P}^{2}}(3 d) \otimes \omega_{\mathbf{P}^{2}}
$$

so, as a sheaf on the ambient space of the $d$-uple embedding of $\mathbf{P}^{2}, \mathcal{F}$ satisfies the duality hypothesis of Corollary 2.3. Furthermore, the given presentation of $\mathcal{F}$ shows that $\mathcal{F}$ is $(d-1)$-regular as a sheaf on $\mathbf{P}^{2}$, and thus it is 0-regular on the ambient space of the $e$-uple embedding for any $e \geq d-1$. Thus Corollary 2.3 shows that $\mathcal{F}$ is an Ulrich sheaf on the $d$-uple embedding.

The Betti diagram of the Tate resolution of a rank 2 sheaf $\mathcal{F}$ satisfying the hypothesis of Proposition 5.9 is given just after Theorem 5.1. Instead of specifying $\alpha$, we could define $\mathcal{F}$ by giving the $(d-1) \times 2(d-2)$ matrix $\beta$ of linear forms over $E$ that occurs at the end of the middle strand of the Tate resolution. For the choice of $\alpha$ above we get

$$
\beta=\left(\begin{array}{ccccccccc}
e_{0} & e_{1} & 0 & 0 & & \ldots & & 0 \\
e_{1} & e_{2} & e_{0} & e_{1} & & & & \\
0 & 0 & e_{1} & e_{2} & & & & \vdots \\
\vdots & & & & \ddots & & & 0 \\
& & & & & & e_{0} & e_{1} \\
0 & & & \ldots & & 0 & e_{1} & e_{2}
\end{array}\right),
$$

and the vector bundle $\mathcal{E}$ has a conic of maximal order jumping lines. One can show by semi-continuity that $\beta$ can be taken to be any sufficiently general $(d-1) \times 2(d-2)$ matrix of linear forms over $E$, but unlike for the matrix $\alpha$, we do not know how to recognize when $\beta$ is sufficiently general to give rise to a Tate resolution of the right form.

\section{Bundles on $\mathbf{P}^{3}$.}

Proposition 5.10. Suppose $d \geq 2$. There exist rank 2 Ulrich sheaves for the d-uple embedding of $\mathbf{P}^{3}$ if and only if $d \not \equiv 0(\bmod 3)$.

Proof. By [Hartshorne and Hirschowitz 1982] for any given $c_{2}$ there exist rank 2 vector bundles $\mathcal{F}$ with $c_{1}=0$ and natural cohomology on $\mathbf{P}^{3}$, i.e., for each twist $t$ the cohomology groups $\mathrm{H}^{i}(\mathcal{F}(t)) \neq 0$ for at most one $i$. The Hilbert polynomial 
$\chi(\mathcal{F}(t))=\frac{1}{6}(t+2)\left(t^{2}+4 t+3-3 c_{2}\right)$ has three integral roots if and only if $1+3 c_{2}$ is a square. Thus for $d \not \equiv 0(\bmod 3)$ and $c_{2}=\left(d^{2}-1\right) / 3$ the sheaf $\mathcal{F}(d-2)$ is Ulrich for the d-uple embedding. The converse follows from Corollary 5.3 .

Remark. The bundles $\mathcal{F}$ in the proof of the proposition are called instanton bundles Tikhomirov 1997] because they satisfy the instanton conditions

$\mathcal{F}$ is stable of rank $2, c_{1}(\mathcal{F})=0$ and $\mathrm{H}^{1}(\mathcal{F}(-2))=0$.

Equivalently their linear monad $\mathbf{L}(\mathcal{F})$ has shape

$$
0 \rightarrow \mathcal{O}(-1)^{c_{2}} \rightarrow \mathcal{O}^{2 c_{2}+2} \rightarrow \mathcal{O}(1)^{c_{2}} \rightarrow 0
$$

Except for the 2-uple embedding $\left(c_{2}=1\right)$, it is an open problem to find an explicit expression for rank 2 Ulrich sheaves of this type.

For the 2-uple embedding the rank 2 Ulrich sheaf is essentially unique:

Proposition 5.11. If $\mathcal{E}$ is the null correlation bundle on $\mathbf{P}^{3}$, then the pushforward of $\mathcal{F}:=\mathcal{E}(-2)$ is, up to automorphisms of $\mathbf{P}^{3}$, the unique rank 2 Ulrich sheaf on the 2-uple embedding on $\mathbf{P}^{3}$.

Proof. By Theorem 5.1, $\mathcal{F}$ is a rank 2 Ulrich sheaf if and only if the Betti diagram of the Tate resolution of $\mathcal{F}$ has the form

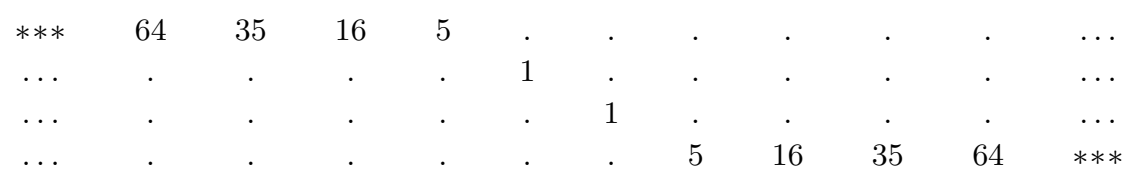

with Hilbert polynomial $\chi \mathcal{F}(t)=\frac{1}{3}(t+2)(t+4)(t+6)$. (Here and henceforward, we replace each zero in a Betti diagram by a "." to improve legibility.) By Okonek et al. 1980, II.3.2, Example 6], the null correlation bundle is determined (up to twist) by its intermediate cohomology $\mathcal{F}$ and the choice of a nondegenerate 2 -form (the 2 -form is visible here as the map in the middle of the Tate resolution). Thus $\mathcal{F}$ must be a twist of the null correlation bundle; the twist is determined by a comparison of Hilbert polynomials.

By Corollary 5.3 there is no rank 2 bundle on $\mathbf{P}^{3}$ that is an Ulrich sheaf for the 3-uple embedding. Corollary 5.7 gives a homogeneous bundle of rank 9 . The following example gives a whole family of rank 3 Ulrich bundles for this case. These bundles give determinantal Bézout formulas for the cube of the resultant of 4 forms of degree 3 in 4 variables.

Example 5.12. [A family of rank 3 vector bundles on $\mathbf{P}^{3}$ which are Ulrich sheaves for the 3-uple embedding]

By Theorem $[5.1 \mathcal{F}$ is an Ulrich sheaf for the 3-uple embedding if and only if the Betti diagram of its Tate resolution has the form 


$\begin{array}{cccccccccccc}\ldots & 81 & 40 & 14 & & . & . & . & . & . & . & . \\ . & . & . & . & 5 & 4 & . & . & . & . & . & . \\ . & . & . & . & . & . & 4 & 5 & . & . & . & . \\ . & . & . & . & . & . & . & . & 14 & 40 & 81 & \ldots\end{array}$

Calculation shows that if we take a sufficiently general $5 \times 4$ matrix over the exterior algebra in 4 variables, then its Tate resolution has this form.

It follows at once from the definitions that a sheaf on $\mathbf{P}^{k}$ becomes weakly Ulrich on the $d$-uple embedding if and only if

$$
\begin{aligned}
& h^{0} \mathcal{F}(-2 d)=0 ; \\
& h^{i} \mathcal{F}((-i-2) d)=0=h^{i} \mathcal{F}((-i+1) d), \quad 0<i<k-1 ; \text { and } \\
& h^{k} \mathcal{F}((-k+1) d)=0 .
\end{aligned}
$$

From the form of the cohomology diagram of the "null correlation bundle" on $\mathbf{P}^{3}$ given in the proof of Proposition 5.11] we see that a twist of this bundle becomes weakly Ulrich on each $d$-uple embedding and thus gives a Pfaffian Stiefel formula for the resultant of 4 forms in 4 variables of any degree. For any $d \geq 2$ the corresponding 2-term complex on $\mathbf{G}\left(4, \mathrm{H}^{0} \mathcal{O}_{\mathbf{P}^{3}}(d)\right)$ has the form

$$
0 \rightarrow \mathcal{O}(-1)^{b} \oplus U^{a} \rightarrow \mathcal{O}^{b} \oplus\left(\Lambda^{3} U\right)^{a} \rightarrow 0
$$

with $a=d\left(d^{2}-4\right) / 3$ and $b=2 d\left(4 d^{2}-4\right) / 3$.

\section{Bundles on $\mathbf{P}^{4}$.}

Example 5.13. The Horrocks-Mumford bundle on $\mathbf{P}^{4}$ has rank 2 and Tate resolution

$$
\begin{array}{ccccccccccccc}
\ldots & 100 & 35 & 4 & . & . & . & . & . & . & . & . & . \\
. & . & 2 & 10 & 10 & 5 & . & . & . & . & . & . & . \\
. & . & . & . & . & . & 2 & . & . & . & . & . & . \\
. & . & . & . & . & . & . & 5 & 10 & 10 & 2 & . & . \\
. & . & . & . & . & . & . & . & . & 4 & 35 & 100 & \ldots
\end{array}
$$

It gives rise to Pfaffian Stiefel formulas for $d=4,6,8$.

Example 5.14. Suppose again that $k=4$, and take $d=2$. By Corollary 5.3 any Ulrich sheaf on the 2-uple embedding of $\mathbf{P}^{4}$ has rank divisible by 8 . Consider a general map $E^{3} \rightarrow E^{5}(-2)$. Its Tate resolution is

$\begin{array}{ccccccccc}\ldots & 128 & 35 & . & . & . & . & . & . \\ . & . & . & 5 & . & . & . & . & . \\ . & . & . & . & 3 & . & . & . & . \\ . & . & . & . & . & 5 & . & . & . \\ . & . & . & . & . & . & 35 & 128 & \ldots\end{array}$

This gives a rank 8 Ulrich sheaf.

\section{Surfaces}

Throughout this section, $X$ denotes a nonsingular projective surface over $K$, and we assume that $K$ has characteristic 0 . We study Ulrich sheaves on $X$. We write $H$ for a hyperplane divisor and $K_{X}$ for a canonical divisor on $X$. 
In general it is rare to find an Ulrich line bundle on a surface; for example, it is easy to see that there is none on the $d$-uple embedding of $\mathbf{P}^{2}$ when $d>1$. Thus we turn to rank 2 bundles. If $\mathcal{F}$ is a rank 2 vector bundle on $X$ such that $c_{1} \mathcal{F}=3 H+K_{X}$ and $\mathcal{F}$ is 0 -regular, then $\mathcal{F}$ is Ulrich by Corollary 2.3. We will call such a rank 2 bundle a special rank 2 Ulrich bundle; it gives rise to a Bézout expression for the Chow form of $X$ as a Pfaffian.

Many surfaces have no rank 2 Ulrich bundles. For example, one can see by considering the dimensions of the families that the general surface $X$ of degree $d \geq 16$ in $\mathbf{P}^{3}$ is not defined by the Pfaffian of a $2 d \times 2 d$ skew-symmetric linear matrix Beauville 2000. Thus such a surface has no special rank 2 Ulrich bundle, and because Pic $X=\mathbf{Z}$ for a general surface, every rank 2 Ulrich sheaf would be special.

We are particularly interested in the case when $X$ is a blow-up of $\mathbf{P}^{2}$ at a set of points $E=\left\{p_{1}, \ldots, p_{e}\right\}$. Write $L$ for the pull-back to $X$ of the class of a line on $\mathbf{P}^{2}$ and $E_{i}$ for the preimage of $p_{i}$. If we take an embedding of $X$ corresponding to a linear series $\left|d L-\sum_{1}^{e} E_{i}\right|$, then the vanishing of the Chow form is the condition for 3 forms of degree $d$ that vanish on $E$ to vanish at a further common point. (This has also been called the "residual resultant", studied in the case of complete intersections in Busé et al. 2001.) We are able to find rank 2 Ulrich sheaves, corresponding to Pfaffian Bézout formulas for the resultant, if the ideal of the set of base points $E$ is generated in degree $<d$.

Remark 6.1. Stieffel resultant formulas for toric surfaces were obtained by A. Khetan, who identifies a class of weakly Ulrich line bundles on such surfaces and finds explicit formulas by constructing syzygies over the exterior algebra, using the method illustrated in Proposition 4.2 See Khetan 2002.

Proposition 6.2. $\quad$ (a) Let $C$ be a smooth curve on $X$ of class $3 H+K_{X}$ and let $\mathcal{L}$ be a line bundle on $C$ with

$$
\operatorname{deg} \mathcal{L}=\frac{1}{2} H \cdot\left(5 H+3 K_{X}\right)+2 \chi \mathcal{O}_{X} .
$$

If $\sigma_{0}, \sigma_{1} \in \mathrm{H}^{0}(\mathcal{L})$ define a base point free pencil and $\mathrm{H}^{1} \mathcal{L}\left(H+K_{X}\right)=0$, then the bundle $\mathcal{F}$ defined by the "Mukai exact sequence"

$$
0 \rightarrow \mathcal{F}^{*} \rightarrow \mathcal{O}_{X}^{2} \stackrel{\left(\sigma_{0}, \sigma_{1}\right)}{\longrightarrow} \mathcal{L} \rightarrow 0
$$

is a special rank 2 Ulrich bundle.

(b) Every special rank 2 Ulrich bundle on $X$ can be obtained from a Mukai sequence as in part (a).

Proof. (a) We begin by proving that, under the hypotheses of part (a), the map

$$
\left(\mathrm{H}^{0} \mathcal{O}\left(H+K_{X}\right)\right)^{2} \stackrel{\left(\sigma_{0}, \sigma_{1}\right)}{\longrightarrow} \mathrm{H}^{0} \mathcal{L}\left(H+K_{X}\right)
$$

is an isomorphism. Using Riemann-Roch on $X$ and on $C$ and the given degree of $\mathcal{L}$, we immediately compute $\chi\left(\mathcal{L}\left(H+K_{X}\right)\right)=2 \chi\left(\mathcal{O}_{X}\left(H+K_{X}\right)\right)$ and $\chi\left(\mathcal{L}\left(2 H+K_{X}\right)\right)=$ $2 \chi\left(\mathcal{O}_{X}\left(2 H+K_{X}\right)\right)$. Our hypothesis that $\mathcal{L}\left(H+K_{X}\right)$ is nonspecial implies that $\mathcal{L}\left(2 H+K_{X}\right)$ is also nonspecial. With this and the Kodaira vanishing theorem on $X$, we see that $\chi$ is equal to $\mathrm{h}^{0}$ for all four of these bundles. Thus it suffices to show that the map $(*)$ is injective.

Since $C \sim 3 H+K_{X}$, there is an exact sequence

$$
0 \rightarrow \mathcal{O}_{X}(-2 H) \rightarrow \mathcal{O}_{X}\left(H+K_{X}\right) \rightarrow O_{C}\left(H+K_{X}\right) \rightarrow 0,
$$


from which we see that the restriction map $\mathrm{H}^{0} \mathcal{O}_{X}\left(H+K_{X}\right) \cong \mathrm{H}^{0} \mathcal{O}_{C}\left(H+K_{X}\right)$ is an injection. By the base point free pencil trick there is a left exact sequence

$$
0 \rightarrow \mathrm{H}^{0} \mathcal{L}^{*}\left(H+K_{X}\right) \rightarrow\left(\mathrm{H}^{0} \mathcal{O}_{C}\left(H+K_{X}\right)\right)^{2} \stackrel{\left(\sigma_{0}, \sigma_{1}\right)}{\longrightarrow} \mathrm{H}^{0} \mathcal{L}\left(H+K_{X}\right)
$$

By the adjunction formula $K_{C}=\left.\left(3 H+2 K_{X}\right)\right|_{C}$, so our hypothesis and Serre duality give $0=\mathrm{h}^{1} \mathcal{L}\left(H+K_{X}\right)=\mathrm{h}^{0} \mathcal{L}^{*}\left(2 H+K_{X}\right)$, whence $\mathrm{h}^{0} \mathcal{L}^{*}\left(H+K_{X}\right)=0$ as well. Thus $(*)$ is an injection.

We can now prove that $\mathcal{F}$ is Ulrich. (The conclusion depends only on the Mukai sequence (with the class of $C$ ) and the fact that (*) is an isomorphism). The Mukai sequence implies that $\bigwedge^{2} \mathcal{F}=\mathcal{O}_{X}\left(3 H+K_{X}\right)$, so by Corollary 2.3 it suffices to show that $\mathcal{F}$ is 0 -regular. Twisting the Mukai sequence by $H+K_{X}$ and using the fact that $(*)$ is an isomorphism, together with Kodaira vanishing, we see that $\mathrm{H}^{1}\left(\mathcal{F}^{*}\left(H+K_{X}\right)\right)=\mathrm{H}^{2}\left(\mathcal{F}^{*}\left(H+K_{X}\right)\right)=0$. Serre duality now gives $\mathrm{H}^{1}(\mathcal{F}(-H))$ $=0$ and $\mathrm{H}^{0}(\mathcal{F}(-H))=0$. Since $\bigwedge^{2} \mathcal{F}=\mathcal{O}_{X}\left(3 H+K_{X}\right)$, we have $\mathcal{F}(-H)=$ $\mathcal{F}^{*}\left(2 H+K_{X}\right)$. By Serre duality $\mathrm{h}^{2}(\mathcal{F}(-2 H))=\mathrm{h}^{0}\left(\mathcal{F}^{*}\left(2 H+K_{X}\right)\right)=\mathrm{h}^{0}(\mathcal{F}(-H))=$ 0 , and $\mathcal{F}$ is Ulrich as claimed.

(b) Conversely, if $\mathcal{F}$ is a special Ulrich bundle of rank 2, then two general sections $\tau_{0}, \tau_{1}$ of $\mathcal{F}$ become dependent on a smooth curve $C$ of class $3 H+K_{X}$. The cokernel of the induced map $0 \rightarrow \mathcal{F}^{*} \rightarrow \bigoplus_{1}^{2} \mathcal{O}$ is a line bundle $\mathcal{L}$ on $C$, generated by global sections, so we obtain the Mukai sequence

$$
0 \rightarrow \mathcal{F}^{*} \rightarrow \bigoplus_{1}^{2} \mathcal{O} \stackrel{\left(\sigma_{0}, \sigma_{1}\right)}{\longrightarrow} \mathcal{L} \rightarrow 0
$$

By Serre duality, $\chi\left(\mathcal{F}^{*}\left(H+K_{X}\right)\right)=\chi(\mathcal{F}(-H))$, which is 0 since $\mathcal{F}$ is Ulrich. Thus $\chi\left(\mathcal{L}\left(H+K_{X}\right)\right)=2 \chi\left(O_{X}\left(H+K_{X}\right)\right)$. Applying the Riemann-Roch theorems on $X$ and $C$ again, we obtain the desired formula for the degree of $\mathcal{L}$. The long exact sequence coming from the Mukai sequence together with Kodaira vanishing and the 0 -regularity of $\mathcal{F}$ show that $\mathcal{L}$ is nonspecial.

Corollary 6.3. Let $C$ be a smooth curve of class $3 H+K_{X}$ and let $\mathcal{L}$ be a line bundle on $C$ such that $|\mathcal{L}|$ is a base point free pencil of degree $\operatorname{deg} \mathcal{L}$ $=\frac{1}{2} H \cdot\left(5 H+3 K_{X}\right)+2 \chi \mathcal{O}_{X}$. The conditions of Proposition 6.2 are satisfied if and only if $|\mathcal{L}|$ does not arise as a projection from $\left|\mathcal{O}_{C}\left(2 H+K_{X}\right)\right|$.

Proof. To say that $|\mathcal{L}|$ arises as a projection from $\left|\mathcal{O}_{C}\left(2 H+K_{X}\right)\right|$ means that $\mathrm{H}^{0}\left(\mathcal{L}^{*}\left(2 H+K_{X}\right)\right) \neq 0$. This space is Serre dual to $\mathrm{H}^{1}\left(\mathcal{L}\left(H+K_{X}\right)\right)$.

Remark 6.4. Pencils which arise as projections correspond to codimension 2 planes that are $\frac{7}{2} H \cdot\left(H+K_{X}\right)+K_{X}^{2}-2 \chi \mathcal{O}_{X}$-secant to $C \subset \mathbf{P} H^{0} \mathcal{O}\left(2 H+K_{X}\right)$. Every component of the variety of such secants has dimension at least

$$
\frac{1}{2} H \cdot\left(H-K_{X}\right)+4 \chi \mathcal{O}_{X}-4-K_{X}^{2},
$$

and we might expect equality. On the other hand the variety of pencils $|\mathcal{L}|$ has dimension at least

$$
\rho(\mathcal{L})=2 \operatorname{deg} \mathcal{L}-g_{C}-2=\frac{1}{2} H \cdot\left(H-3 K_{X}\right)+4 \chi \mathcal{O}_{X}-1-K_{X}^{2} .
$$

Thus we would expect the existence of an $\mathcal{L}$ which is not a projection, and thus of a special rank 2 Ulrich bundle, in case $H \cdot K_{X}<3$. 
Resultants of ternary forms with base points. Consider $X=\mathbf{P}^{2}\left(p_{1}, \ldots, p_{e}\right)$ the blow up of the plane in $e$ distinct points and a very ample divisor class $H=$ $d L-\sum_{i=1}^{e} E_{i}$. Here $L$ denotes the class of a line and the $E_{i}$ the exceptional divisors. We first treat forms of degree $d=3$ - that is, Del Pezzo surfaces. In this case the results are immediate from what we have already done.

Corollary 6.5. Suppose that the base field $K$ is algebraically closed. If $X \subset \mathbf{P}^{n}$ is a del Pezzo surface, then $X$ has a special rank 2 Ulrich bundle. Thus there is a Pfaffian Bézout formula for the resultant of 3 ternary cubics with d base points in general position.

Proof. In this case $K_{X}=-H$ and $C \sim 3 H+K_{X}$ is a canonical curve of genus $g=H^{2}+1=n+1$. Any general line bundle of degree

$$
\operatorname{deg} \mathcal{L}=\frac{1}{2} H \cdot\left(5 H+3 K_{X}\right)+2 \chi \mathcal{O}_{X}=g+1
$$

defines a nonspecial pencil. Thus we can apply Proposition 6.2 to get a special rank 2 Ulrich bundle on $X$.

The space of ternary cubics with $d$ general base points has dimension $10-d$, so it suffices to treat the case of seven or fewer points. The linear series of cubics with 7 assigned base points maps the plane two-to-one onto itself, and the condition that three such cubics meet in an extra point is the condition that three lines in the plane meet in a point - a determinantal condition.

For six or fewer assigned base points the resultant is exactly the Chow form of the corresponding del Pezzo surface.

In the case of higher degree forms, our results are less complete:

Theorem 6.6. Let the ground field be infinite. Let $E=\left\{p_{1}, \ldots, p_{e}\right\}$ be a collection of e distinct points in $\mathbf{P}^{2}$ and let $X=\mathbf{P}^{2}\left(p_{1}, \ldots, p_{e}\right)$ be the blow up of $\mathbf{P}^{2}$ in these points, embedded by the linear system $\left|d L-\sum E_{i}\right|$. If the homogenous ideal $I_{E}$ of the points is generated in degree $d-1$, then $X$ has a special rank 2 Ulrich sheaf.

Proof. Let $\eta: X \rightarrow \mathbf{P}^{2}$ be the blow up. By Proposition 6.2 we have to construct a pencil $|\mathcal{L}|$ of degree $\frac{(d-1)(5 d-4)}{2}-e$ on a smooth curve of class $(3 d-3) L-2 \sum_{i} E_{i}$ on $X$ which satisfies $\mathrm{H}^{1} \mathcal{L}\left(H+K_{X}\right)=\mathrm{H}^{1} \mathcal{L}((d-3) L)=0$. Let $C^{\prime}=\eta(C) \subset \mathbf{P}^{2}$ be the plane model, so that $C^{\prime}$ is a curve of degree $3 d-3$ passing doubly through the points $\left\{p_{1}, \ldots, p_{e}\right\}$. Every pencil on $C$ can be written as a pencil of adjoint curves of degree $a$, say, with assigned base points $F=q_{1}+\ldots+q_{f}$ on $C^{\prime}$, that is, a pencil $\left\{\lambda A_{0}+\mu A_{1}\right\} \subset \mathrm{H}^{0}\left(\mathbf{P}^{2}, \mathcal{I}_{E \cup F}(a)\right)$. The pencil of plane curves might have additional base points $G=r_{1}+\ldots+r_{g}$ away from $C^{\prime}$. We have

$$
a^{2}=e+f+g \text {. }
$$

In order that $|\mathcal{L}|$ is not a projection from $\left|2 H+K_{X}\right|$ we need $a>2 d-3$. We choose $a=2 d-2$ so that we can deal with the fewest number of additional points $F$ and $G$.

To make the construction, we will choose $G$ and then the pencil $\left\langle A_{0}, A_{1}\right\rangle$. This will determine $F$. Finally, we will choose $C^{\prime}$ and $\mathcal{L}^{\prime}=\eta_{*} \mathcal{L}$.

Take $G=r_{1}+\ldots+r_{g}$ to be a set of $g=\left(\begin{array}{l}d \\ 2\end{array}\right)$ general points in the plane disjoint from $E$. Because $G$ is general, the ideal $I_{G}$ contains $d$ independent forms of degree $d-1$ and no forms of degree $d-2$. It follows from the Hilbert-Burch Theorem (Eisenbud 1995, 20.4]) $I_{G}$ is generated by the $d-1$ minors of a $d \times(d-1)$ matrix $\varphi_{1}: \mathcal{O}_{\mathbf{P}^{2}}(-1)^{d-1} \rightarrow \mathcal{O}_{\mathbf{P}^{2}}^{d}$ with linear entries. 
Since $I_{E}$ and $I_{G}$ are generated by forms of degree $d-1$, and $E \cap G=\emptyset$, the sheaf $\mathcal{I}_{E \cup G}(2 d-2)$ is globally generated. By Bertini's Theorem we may choose a pencil

$$
A_{0}, A_{1} \in \mathrm{H}^{0}\left(\mathbf{P}^{2}, \mathcal{I}_{E \cup G}(2 d-2)\right)
$$

of forms of degree $a=2 d-2$ vanishing simply at $E \cup G$ and at a set $F$ of $f$ points outside $E \cup G$.

The set of points $E \cup F$ is thus geometrically linked to $G$ by the complete intersection $\left(A_{1}, A_{2}\right)$ in the sense of Peskine and Szpiro 1974]. It was observed by Apery and Gaeta (see, for example, Eisenbud 1995, Proposition 21.24]) that the ideal $I_{E \cup F}=\left(A_{0}, A_{1}\right): I_{G}$ of $E \cup F$ is generated by the $d \times d$ minors of the $d \times(d+1)$ matrix $\varphi_{2}: \mathcal{O}_{\mathbf{P}^{2}}(-1)^{d-1} \oplus \mathcal{O}_{\mathbf{P}^{2}}(-d+1)^{2} \rightarrow \mathcal{O}^{d}$ obtained by bordering the matrix $\varphi_{1}$ with two columns containing the coefficients necessary to express $A_{0}$ and $A_{1}$ as linear combinations of the generators of $I_{G}$.

Let $C^{\prime}$ be the curve defined by a general form of degree $3 d-3$ vanishing doubly along $E$ and simply along $F$. Since this form lies in $I_{E \cup F}$, it can be expressed as a linear combination of the minors of $\varphi_{2}$. Thus it is the determinant of a matrix $\varphi_{3}: \mathcal{O}_{\mathbf{P}^{2}}(-1)^{d-1} \oplus \mathcal{O}_{\mathbf{P}^{2}}(-d+1)^{2} \rightarrow \mathcal{O}_{\mathbf{P}^{2}}^{d+1}$ obtained from $\varphi_{2}$ by adding a column. Since we can think of the entries of $\varphi_{3}$ as general linear forms and general elements of $\left(I_{E}\right)_{d-1}$, we see that $C^{\prime}$ is nonsingular away from $E$ and has only ordinary double points in $E$.

We define $\mathcal{L}^{\prime}$ to be the cokernel of the transpose of $\varphi_{3}$, twisted by $\mathcal{O}_{\mathbf{P}^{2}}(-d+1)$ :

$$
0 \rightarrow \mathcal{O}_{\mathbf{P}^{2}}(-d+1)^{d+1} \stackrel{\varphi_{3}^{t}(-d+1)}{\longrightarrow} \mathcal{O}_{\mathbf{P}^{2}}(-d+2)^{d-1} \oplus \mathcal{O}_{\mathbf{P}^{2}}^{2} \rightarrow \mathcal{L}^{\prime} \rightarrow 0 .
$$

Locally around a point $p_{i}$ of $E$ the sheaf $\mathcal{L}^{\prime}$ is minimally generated by two elements, since $A_{0}$ and $A_{1}$ intersect transversally at $p_{i}$. Thus $\mathcal{L}^{\prime}$ is locally isomorphic to $\eta_{*} \mathcal{O}_{C}$, and we must have $\mathcal{L}^{\prime}=\eta_{*} \mathcal{L}$ for some line bundle $\mathcal{L}$ on $C$.

Since $\mathrm{H}^{0} \mathcal{L}=\mathrm{H}^{0} \mathcal{L}^{\prime}$, the two global sections of $\mathcal{L}^{\prime}$ that are the images of the two global sections of the middle term in the sequence above come from global sections $\sigma_{0}, \sigma_{1}$ of $\mathcal{L}$. We claim that the kernel $\mathcal{F}$ of the map $\mathcal{O}_{X}^{2} \rightarrow \mathcal{L}$ defined by these sections is an Ulrich sheaf on $X$.

As in the proof of Proposition 6.2 it suffices to show that the map $\left(\sigma_{0}, \sigma_{1}\right)$ : $\left(\mathrm{H}^{0} \mathcal{O}_{X}\left(H+K_{X}\right)\right)^{2} \rightarrow \mathrm{H}^{0} \mathcal{L}\left(H+K_{X}\right)$ is an isomorphism. Pushing forward and using

$$
\eta_{*} \mathcal{O}_{X}\left(H+K_{X}\right)=\mathcal{O}_{\mathbf{P}^{2}}(d-3),
$$

we must show that the induced map $\left(\mathrm{H}^{0} \mathcal{O}_{\mathbf{P}^{2}}(d-3)\right)^{2} \rightarrow \mathrm{H}^{0} \mathcal{L}^{\prime}(d-3)$ is an isomorphism. Since the additional generators of $\sum_{m} \mathrm{H}^{0}\left(\mathcal{L}^{\prime}(m)\right)$ are of degree $d-2$, this follows from the sequence defining $\mathcal{L}^{\prime}$.

Corollary 6.7. There exists a Pfaffian Bézout formula for ternary forms of degree $d$ with e assigned base points if the ideal of the points is generated in degree $d-1$.

Remark 6.8. Our computations suggest that the construction of the rank 2 Ulrich sheaf above, and hence the construction of a Bézout formula for forms with base points, works for a set of points $E$ even under the weaker hypothesis that $I_{E}$ is generated in degree $d$. For example, if $E$ consists of $e \leq\left(\begin{array}{c}d+2 \\ 2\end{array}\right)-6$ general points, there should be plenty of room to arrive at a nodal $C^{\prime}$ in the construction. 


\section{Appendix: Homomorphisms and extensions Between the Bundles $\bigwedge^{p} U$ ON THE GRASSMANNIAN \\ BY JERZY WEYMAN}

In this appendix we will prove part (b) of Proposition 1.1 and also prove a complementary statement about the higher cohomology.

Theorem 6.9. Let $\mathbf{G}_{l}$ be the Grassmannian of codimension l planes in a vector space $W$ with dual $V=W^{*}$ over a field $K$ of arbitrary characteristic, and let $U$ be the tautological l-sub-bundle of $W \times \mathbf{G}_{l}$. For $0 \leq p, q, \leq l$ we have

$$
\operatorname{Hom}\left(\bigwedge^{q} U, \bigwedge^{p} U\right)= \begin{cases}0, & \text { if } p>q \\ \bigwedge^{q-p} V, & \text { otherwise. }\end{cases}
$$

Moreover $\operatorname{Ext}^{i}\left(\bigwedge^{q} U, \bigwedge^{p} U\right)=0$ for $i>0$ and all $p, q$.

In characteristic 0 these statements follow from Borel-Weil-Bott theory Jantzen 1987.

Let $\mathrm{GL}=\mathrm{GL}_{K}(W)$ be the general linear group. We write $Q$ for the tautological quotient bundle $Q=W / U$ on $\mathbf{G}_{l}$. If $\lambda=\left(\lambda_{1}, \ldots, \lambda_{v}\right)$ is a nonincreasing sequence of positive integers (a highest weight for GL), then we write $L_{\lambda} W$ for the Schur module corresponding to the highest weight $\lambda$. We may extend this notation to any nonincreasing sequence of integers $\lambda$ (dominant integral weight) using the formula $L_{\lambda} W=L_{\mu^{\prime}} W \otimes\left(\bigwedge^{v} W\right)^{\otimes \lambda_{v}}$ where $\mu^{\prime}$ is the partition conjugate to $\mu=\left(\lambda_{1}-\right.$ $\left.\lambda_{v}, \ldots, \lambda_{v-1}-\lambda_{v}, 0\right)$. The proof of Theorem 6.9 rests on the following facts:

Lemma 6.10. The tensor product $\bigwedge^{p} U \otimes \bigwedge^{q} U^{*}$ has a filtration with the associated graded object

$$
\bigoplus_{\substack{a+b=p-q \\ 0 \leq a \leq p, 0 \leq b \leq q, a+b \leq l}} L_{\left(1^{a}, 0^{l-a-b},(-1)^{b}\right)} U .
$$

Lemma 6.11. (a) If $a>0$, then all cohomology groups of the vector bundles $L_{\left(1^{a}, 0^{l-a-b},(-1)^{b}\right)} U$ are zero.

(b) All higher cohomology groups of the bundle $L_{\left(0^{l-b},(-1)^{b}\right)} U$ are zero and

$$
H^{0}\left(\mathbf{G}_{l}, L_{\left(0^{l-b},(-1)^{b}\right)} U\right)=\bigwedge^{b} W^{*} .
$$

Proof of Lemma 6.10, It is a standard fact on good filtrations [Donkin 1985] that the tensor product of Schur modules has a filtration with associated graded module being a direct sum of Schur modules. The multiplicities of the Schur modules occurring are the same as in characteristic zero, and we can get the result by the Littlewood-Richardson rule, using the isomorphism $\bigwedge^{q} U^{*}=\bigwedge^{l-q} U \otimes \bigwedge^{l} U^{*}$.

Proof of Lemma 6.11. Let $\lambda=\left(\lambda_{1}, \ldots, \lambda_{v}\right)$ be an $v$-tuple of integers. Consider the full flag variety and the tautological subbundles $U_{i}$ of rank $i$ on it. We denote by $\mathcal{L}(\lambda)=\bigotimes_{1 \leq i \leq v}\left(U_{i} / U_{i-1}\right)^{-\lambda_{i}}$ the line bundle on the full flag variety GL/B, where $B$ is the Borel subgroup. Then:

Lemma 6.12. (a) If $\lambda$ is a dominant integral weight, then the higher cohomology groups of $\mathcal{L}(\lambda)$ vanish and

$$
H^{0}(\mathrm{GL} / B, \mathcal{L}(\lambda))=L_{\lambda} W \text {. }
$$


(b) Let us assume that for some $i$ we have $\lambda_{i}=\lambda_{i-1}+1$. Then all cohomology groups of $\mathcal{L}(\lambda)$ vanish.

To prove part (a) of Lemma 6.11 we consider the natural projection $\eta:$ GL/ $B \rightarrow$ $\mathbf{G}_{l}$. We observe that by Kempf's Vanishing Theorem in the relative setting Jantzen 1987, we have $L_{\left(1^{a}, 0^{l-a-b},(-1)^{b}\right)} U=\eta_{*}\left(\mathcal{L}\left(0^{v-l}, 1^{a}, 0^{l-a-b},(-1)^{b}\right)\right)$ with higher direct images

$$
\mathcal{R}^{i} \eta_{*}\left(\mathcal{L}\left(0^{v-l}, 1^{a}, 0^{l-a-b},(-1)^{b}\right)\right)
$$

being zero for $i>0$. Since by Lemma 6.12 (b) we know that all cohomology groups of $\mathcal{L}\left(0^{v-l}, 1^{a}, 0^{l-a-b},(-1)^{b}\right)$ are zero, by the spectral sequence of the composition we are done. Now part (b) of Lemma 6.11 follows from part (a) of Lemma6.12.

Proof of Lemma 6.12 Part (a) is Kempf's Vanishing Theorem (see Kempf 1976 or [Haboush 1980]). Part (b) follows from the following consideration. Let $P(i)$ be a parabolic subgroup such that the corresponding homogeneous space is a flag variety of flags of dimensions $(1,2, \ldots, i-1, i+1, \ldots, v-1, v)$. The projection $\rho: \mathrm{GL} / B \rightarrow$ $\mathrm{GL} / P(i)$ allows us to identify $\mathrm{GL} / B$ with the projectivization $\mathbf{P}\left(U_{i+1} / U_{i-1}\right)$. The bundle $\mathcal{L}(\lambda)$ is of the form $\rho^{*}(\mathcal{M}) \otimes \mathcal{O}_{\mathbf{P}\left(U_{i+1} / U_{i-1}\right)}(-1)$ because all the factors in the definition of $\mathcal{L}\left(\lambda_{1}, \ldots, \lambda_{v}\right)$ except of the $i$-th and $i+1$-st are induced from GL/P $(i)$. Therefore by Serre's Theorem (in the relative setting) and by the projection formula we see that all higher direct images $\mathcal{R}^{i} \rho_{*}\left(\mathcal{L}\left(\lambda_{1}, \ldots, \lambda_{v}\right)\right)$ are zero. This implies part (b).

\section{ACKNOWLEDGEMENTS}

The authors are grateful to Hans-Christian v. Bothmer, Wolfram Decker, Joe Harris, Jürgen Herzog, Michael Kapranov, Bernd Sturmfels, and Jerzy Weyman for discussions of various parts of this material. Finally, this paper owes much to experiments made with the computer algebra system Macaulay2 Grayson and Stillman 1993- ; our thanks to Dan Grayson and Mike Stillman for writing it and for their support in using it for this project.

\section{REFERENCES}

B. Angéniol and M. Lejeune-Jalabert: Calcul différentiel et classes caractéristiques en géométrie algébrique. Travaux en Cours 38, Hermann, Paris, 1989. MR 90h:14004

J. Backelin and J. Herzog: On Ulrich-modules over hypersurface rings. Commutative algebra (Berkeley, CA, 1987), 63-68, Math. Sci. Res. Inst. Publ., 15, Springer, New York, 1989. MR 90i: 13006

A. Beauville: Determinantal hypersurfaces. Dedicated to William Fulton on the occasion of his 60th birthday. Mich. Math. J. 48 (2000) 39-64. MR 2002b:14060

A. Beilinson: Coherent sheaves on $\mathbf{P}^{n}$ and problems of linear algebra. Funct. Anal. and its Appl. 12 (1978) 214-216. (Trans. from Funkz. Anal. i. Ego Priloz 12 (1978) 68-69.) MR 80c:14010b

I.N. Bernstein, I.M. Gel'fand and S.I. Gel'fand: Algebraic bundles on $\mathbf{P}^{n}$ and problems of linear algebra. Funct. Anal. and its Appl. 12 (1978) 212-214. (Trans. from Funkz. Anal. i. Ego Priloz 12 (1978) 66-67.) MR 80c:14010a

E. Bézout: Théorie générale des équation algébriques, Pierres, Paris 1779.

J. Brennan, J. Herzog, and B. Ulrich: Maximally generated Cohen-Macaulay modules. Math. Scand. 61 (1987) 181-203. MR 89j:13027

R.-O. Buchweitz and F.-O. Schreyer: Complete intersections of two quadrics, hyperelliptic curves and their Clifford Algebras, manuscript, 2002.

R.-O. Buchweitz, D. Eisenbud and J. Herzog: Cohen-Macaulay modules on quadrics, In Singularities, representation of algebras, and vector bundles (Lambrecht, 1985), Springer- Lecture Notes in Math. 1273 (1987) 96-116. MR 89g:13005 
L. Busé, M. Elkadi and B. Mourrain: Resultant over the residual of a complete intersection. J. Pure Appl. Algebra 164, No.1-2, 35-57 (2001). MR 2002h:13042

C. D'Andrea: Macaulay style formulas for sparse resultants. Trans. Am. Math. Soc. 354, No.7, 2595-2629 (2002). MR 2003a:13032

C. D'Andrea and A. Dickenstein: Explicit formulas for the multivariate resultant. J. Pure Appl. Algebra 164, No.1-2, 59-86 (2001). MR 2002g:13060

A. Cayley: On the theory of elimination. Cambridge and Dublin Mathematical J. 3 (1848) 116120.

S. Donkin: Rational representations of algebraic groups. Tensor products and filtration. Lecture Notes in Math. 1140. Springer, Berlin, 1985. MR 87b:20054

D. Eisenbud: Linear sections of determinantal varieties. American J. Math. 110 (1988) 541-575. MR 89h:14041

D. Eisenbud: Commutative Algebra with a View Toward Algebraic Geometry. Springer Verlag, 1995. MR 97a:13001

D. Eisenbud and S. Goto: Linear free resolutions and minimal multiplicity. J. Alg. 88 (1984) 89-133. MR 85f:13023

D. Eisenbud, G. Fløystad and F.-O. Schreyer: Sheaf cohomology and free resolutions over the exterior algebra. Preprint (2001), arXiv.org/abs/math.AG/0104203.

W. Fulton: Intersection Theory. Springer, New York, 1984. MR 85k:14004

W. Fulton: Young tableaux : with applications to representation theory and geometry. London Mathematical Society student texts 35, Cambridge University Press 1997. MR 99f:05119

I. M. Gelfand, M. Kapranov, and A. Zelevinsky: Discriminants, resultants, and multidimensional determinants. Birkhäuser, Boston, 1994. MR 95e:14045

D. Grayson and M. Stillman: Macaulay2. Available online at http://www.math.uiuc.edu/ Macaulay2/.

W. Haboush: A short proof of the Kempf vanishing theorem. Invent. Math. 56, 109-112 (1980). MR 81g:14008

D. Hanes: Special condition on maximal Cohen-Macaulay modules, and applications to the theory of multiplicities. Thesis, Michigan, 2000.

R. Hartshorne: Algebraic Geometry. Springer Verlag, 1977. MR 57:3116

R. Hartshorne and A. Hirschowitz: Cohomology of the general instanton bundle. Ann. scient. Éc.

Norm. Sup. a série 15 (1982) 365-390. MR 84c:14011

G. Horrocks: Vector bundles on the punctured spectrum of a local ring. Proc. Lond. Math. Soc., III. Ser. 14, 689-713 (1964). MR 30:120

G. Horrocks, D. Mumford: A rank 2 vector bundle on $\mathbf{P}^{4}$ with 15,000 symmetries. Topology 12 (1973) 63-81. MR 52:3164

B. Iverson: Cohomology of sheaves. Springer-Verlag, New York, 1986.

J. C. Jantzen: Representations of algebraic groups. Pure and Applied Mathematics, 131, Academic Press, Boston, MA, 1987. MR 89c:20001

J.-P. Jouanolou: Aspects invariants de l'élimination. Adv. Math. 114 (1995) 1-174. MR 96m:14001

G. R. Kempf: Linear systems on homogeneous spaces. Ann. of Math., II. Ser. 103, 557-591 (1976). MR 53:13229

A. Khetan: Determinantal Formula for the Chow Form of a Toric Surface, In ISSAC Proceedings, 145-150, ACM 2002.

M. Kline: Mathematical thought from ancient to modern times. Oxford University Press, New York 1972. MR 57:12010

F. Knudsen and D. Mumford: The projectivity of the moduli space of stable curves I: Preliminaries on "det" and "div". Math. Scand. 39 (1976) 19-55. MR 55:10465

G. W. Leibniz: Mathematische Schriften, herausgegeben von C. I. Gerhardt, Letter to l'Hospital 28 April 1693, volume II, p. 239; Vorwort volume VII, pp. 5. Georg Olms Verlag Hildesheim, New York 1971. MR 25:4979a, MR 25:4979g

Ch. Okonek, M. Schneider, and H. Spindler: Vector Bundles on Complex Projective Spaces. Birkhäuser, Boston 1980. MR 81b:14001

C. Peskine and L. Szpiro: Liaison des variétés algébriques I. Invent. Math. 26 (1974) 271-302. MR 51:526

R. Stanley: Theory and application of plane partitions. Studies in Appl. Math. 1 (1971) 167-187 and 259-279. MR 48:3754 
R. G. Swan: K-theory of quadric hypersurfaces. Ann. of Math. 122 (1985) 113-153. MR 87g:14006

J. J. Sylvester: A method of determining by mere inspection the derivatives from two equations of any degree. Philosophical Magazine XVI (1840) 132-135. Memoir on the dialytic method of elimination. Part I. Philsophical Magazine XXI (1842) 534-539. Reprinted in: The collected Mathematical Papers of James Joseph Sylvester, Vol. I. Chelsea New York 1973, reprint of the Cambridge 1904 edition.

A. A. Tikhomirov: The main component of the moduli space of mathematical instanton vector bundles on $\mathbf{P}^{3}$. Journal of the Mathematical Sciences 86 (1997) 3004-3087. (Translation from the Russian of Itogi Nauki i Tkhniki. Seriya Sovremennaya Matematika i Ee Prilozheniya. Tematischeskie Obzory, Vol 25, Algebraic Geometry-2, 1995.) MR 99e:14012

B. Ulrich: Gorenstein rings and modules with high numbers of generators. Math. Z. 188 (1984) 23-32. MR 85m:13021

V. Vinnikov: Complete description of determinantal representations of smooth irreducible curves. Linear Algebra Appl. 125 (1989), 103-140. MR 90m:14028

J. Weyman and A. Zelevinsky: Determinantal formulas for multigraded resultants. J. Alg. Geom. 3 (1994) 569-598. MR 95j:14074

Department of Mathematics, University of California, Berkeley, Berkeley, CaliFORNIA 94720

E-mail address: eisenbud@math.berkeley.edu

Mathematik und Informatik, Geb. 27, Universität des SaArlandes, D-66123 SaARBRÜCKEN, GERMANY

E-mail address: schreyer@math.uni-sb.de

Department of Mathematics, Northeastern University, Boston, Massachusetts 02115

E-mail address: weyman@neu.edu 